Juan Garrido, Francisco Vázquez, Fernando Morilla, An extended approach of inverted decoupling, Journal of Process Control, Volume 21, Issue 1, January 2011, Pages 55-68, ISSN 0959-1524, http://dx.doi.org/10.1016/j.jprocont.2010.10.004.

(http://www.sciencedirect.com/science/article/pii/S0959152410001903)

\title{
AN EXTENDED APPROACH OF INVERTED DECOUPLING
}

\author{
Juan Garrido $^{\mathrm{a}^{*}}$, Francisco Vázquez ${ }^{\mathrm{a}}$, Fernando Morilla ${ }^{\mathrm{b}}$ \\ ${ }^{a}$ Department of Computer Science and Numerical Analysis, University of Córdoba, \\ Campus de Rabanales, 14071, Córdoba, Spain \\ *Fax: (+34)957218729; e-mail: juan.garrido@uco.es \\ ${ }^{b}$ Department of Computer Science and Automatic Control, UNED, Juan del Rosal 16, \\ 28040, Madrid, Spain
}

\begin{abstract}
This paper presents an extension of the inverted decoupling approach that allows for more flexibility in choosing the transfer functions of the decoupled apparent process. In addition, the expressions of the inverted decoupling are presented for general nxn processes, highlighting that the complexity of the decoupler elements is independent of the system size. The realizability conditions are stated in order to select a proper configuration, and the different possible cases for each configuration are shown. Comparisons with other works demonstrate the effectiveness of this methodology, through the use of several simulation examples and an experimental lab process.
\end{abstract}

Keywords: decoupling control, inverted decoupling, simplified decoupling, PID control.

\section{Introduction}

Multi-input multi-output (MIMO) processes consist of several measurement and control signals, and there are often complicated couplings between them, which can cause difficulties in feedback controller design. Control engineers traditionally solve these problems using single-loop PID controllers because they can be easily understood and implemented [1]. These decentralized approaches have evolved through years of experience, and they are adequate when the interactions in different channels of the process are modest [2-3]. Nevertheless, when interactions are significant, the decoupling is often treated inefficiently, e.g., by detuning control loops. In these cases, a full matrix controller (centralized control) is advisable.

There are two approaches of centralized control: a pure centralized strategy [4-9] or a decoupling network combined with a diagonal decentralized controller [10-14]. A decentralized control system with a decoupling matrix can be designed by combining a diagonal controller $\mathrm{C}(\mathrm{s})$ with a block compensator $\mathrm{D}(\mathrm{s})$ in such a way that the controller sees the apparent process $G(s) \cdot D(s)$ as a set of $n$ completely independent processes. The essence of decoupling is the imposition of a calculation net that cancels the existing process interaction, allowing for independent control of the loops. Although model predictive control (MPC) is becoming the standard technique to solve multivariable 
control problems in the process industry, several authors $[6,7,12]$ claim that when MPC is used today, it is mostly used on a higher level to give setpoints to the PID controllers that are operating on the basic level. They are operating in a supervisory mode with sampling times that are longer than in the PID controllers at the lower level. And there are some difficulties in dealing with the interaction at the MPC level because the bandwidths of the MPC loops are limited. Therefore, the centralized control using PID controllers and decoupler networks is an interesting strategy in the process industry.

The theory of decoupling control has been addressed in the literature [15-18]. Some decoupling schemes are static [19], and others are dynamic [6], [13], [14], [20]. Most of these methodologies focus on systems with two inputs and two outputs (TITO systems).

Most decoupling approaches use a conventional decoupling scheme in which the process inputs are derived by a time-weighted combination of feedback controller outputs (Figure 1). In this case, the design of the decoupler network for an nxn process is obtained from (1), generally specifying n elements of the decoupler $D(s)$ or the $n$ desired transfer functions of the apparent process Q(s). The most extended forms of conventional decoupling were termed ideal and simplified decoupling in [10]. This approach has received considerable attention in both control theory and industrial practice for several decades.

\section{INSERT HERE FIGURE 1}

$$
D(s)=G^{-1}(s) \cdot Q(s)
$$

In ideal decoupling, the goal is to make the apparent processes as simple as the diagonal elements of the process matrix G(s). The main inconveniences of this method are the complexity of the decoupler elements (2) and realizability problems.

$D(s)=\frac{\operatorname{Adj}(G(s))}{|G(s)|} \cdot G_{\text {diag }}(s)$

On the other hand, with simplified decoupling, $n$ elements of the decoupler, generally the diagonal ones, are set to unity. Decoupler network design is easier, but the complexity of the apparent process (3) is greater since it consists of the determinant of $\mathrm{G}(\mathrm{s})$ and the $\mathrm{g}_{\mathrm{ii}}(\mathrm{s})$ cofactor, $\mathrm{G}^{\mathrm{ii}}(\mathrm{s})$.

$q_{i}(s)=\frac{|G(s)|}{G^{i i}(s)}$

An alternative means of decoupling, called inverted decoupling, derives a process input as a time-weighted combination of one feedback controller output and the other process inputs. It is rarely mentioned in the literature [11], [21], [22], [23], and in those cases, it is only applied to TITO processes using the scheme depicted in Figure 2. In this case, it is possible to keep the same apparent process of ideal decoupling while using the simple decoupler elements of simplified decoupling [11]. 
In [21], a comparative study of simplified, ideal and inverted decoupling is presented, and the practical advantages of inverted decoupling are mentioned. Chen and Zhang [23] improved upon the inverted decoupling technique for a class of stable linear multivariable processes with multiple time delays and non-minimum-phase zeros.

\section{INSERT HERE FIGURE 2}

Nevertheless, the authors have not found any published works in which inverted decoupling is applied to processes that are larger than a 2x2 system. In this work, the formulation of a general inverted structure for decoupling of nxn processes is presented. It is shown that the complexity of the transfer functions of the decoupler elements and of the decoupled equivalent open loop process is independent of the system size. In conventional decouplings, when the input and output number of the square system increases, the complexity of the decoupler elements or the decoupled process is increased as well, and so they are more difficult to implement.

In addition, this formulation is carried out using decoupler elements that differ from unity, in such a way that the decoupled apparent processes can be specified with more flexibility. The paper is structured as follows. Section 2 presents the general formulation of inverted decoupling for nxn processes and the different possible configurations. Furthermore, a study focused on TITO processes is carried out in order to show all possible cases. The expressions for 3x3 processes are also obtained. In Section 3, the realizability conditions are stated and some rules are suggested for selecting a proper configuration. In Section 4, the performance of the proposed decoupling methodology is tested and compared with other methods using several simulation examples and a real quadruple tank process. Finally, conclusions are summarized in Section 5.

\section{Inverted decoupling for nxn processes}

Currently, most papers in the literature regarding inverted decoupling only apply the approach to 2x2 processes (Figure 2), and only the expressions for this case are presented. In this section, the formulation for the general case of nxn processes is calculated, and then, the equations for the cases $n=2$ and $n=3$ are given.

In order to study inverted decoupling for a square process with $\mathrm{n}$ inputs and $\mathrm{n}$ outputs, the use of a matrix representation is proposed as shown in Figure 3. There are $n$ elements of the decoupler ( $\mathrm{Dd}(\mathrm{s})$ matrix), which try to directly connect the decoupler inputs "m" with the process inputs " $u$ ", while the rest of the elements (Do(s) matrix) feedback the input process "u" toward the decoupler inputs in order to decouple the system.

\section{INSERT HERE FIGURE 3}

The whole decoupler $\mathrm{D}(\mathrm{s})$ is split into two matrices: a matrix $\mathrm{Dd}(\mathrm{s})$ in the direct path (between controller outputs c and process inputs u) and a matrix Do(s) in a feedback loop (between process inputs u and controller outputs c). The $\mathrm{Dd}(\mathrm{s})$ matrix must have only n non-zero elements, since there must be only a direct connection for each process input. Note that these relationships are not required in the Do(s) matrix. Additionally, since the signal flow direction in $\operatorname{Do}(\mathrm{s})$ is opposite that of $\operatorname{Dd}(\mathrm{s})$, the corresponding elements of $\mathrm{Do}(\mathrm{s})$ that must equal zero are the transpose non-zero elements of $\mathrm{Dd}(\mathrm{s})$. 
For instance, in a $3 \times 3$ process, if element $\operatorname{Dd}(3,1)$ is specified as a direct path between $\mathrm{u}_{3}$ and $\mathrm{c}_{1}$, there will not be feedback from $\mathrm{u}_{3}$ toward $\mathrm{c}_{1}$, and thus, the element $\operatorname{Do}(1,3)$ must be zero.

Following the decoupler representation given in Figure 3, the expression of the whole decoupler matrix $\mathrm{D}(\mathrm{s})$ is obtained as follows:

$$
\mathrm{D}(\mathrm{s})=\operatorname{Dd}(\mathrm{s}) \cdot(\mathrm{I}-\mathrm{Do}(\mathrm{s}) \cdot \operatorname{Dd}(\mathrm{s}))^{-1}
$$

The transfer function matrix $\mathrm{D}(\mathrm{s})$ of conventional decoupling is related to the inverted decoupling structure according to (4). As it is a complex expression, it is easier to work with its inverse, which is very simple, as follows:

$$
\mathrm{D}^{-1}(\mathrm{~s})=(\mathrm{I}-\mathrm{Do}(\mathrm{s}) \cdot \operatorname{Dd}(\mathrm{s})) \cdot \operatorname{Dd}^{-1}(\mathrm{~s})=\operatorname{Dd}^{-1}(\mathrm{~s})-\operatorname{Do}(\mathrm{s})
$$

Inverting equation (1) and substituting it into (5), the following expression is obtained:

$$
\operatorname{Dd}^{-1}(s)-\operatorname{Do}(s)=Q^{-1}(s) \cdot G(s)
$$

This last expression can be used to calculate the elements of the inverted decoupling. Its main advantage is its simplicity, regardless of the size of the system, because the Q(s) matrix is chosen to be diagonal and the resulting subtraction of the inverse of $\operatorname{Dd}(\mathrm{s})$ and Do(s) is a transfer matrix with only one element to be calculated in each position.

Note that $\operatorname{Dd}(\mathrm{s})$ has to be non-singular because it is inverted, and therefore, when its elements are chosen, only one element in each row and column can be selected. Consequently, for an nxn process there are only $n$ ! possible choices of $\operatorname{Dd}(s)$.

To name these possibilities, the authors propose a notation in which the indicated number corresponds to the column with the selected element. For instance, in a 3x3 process, configuration 1-2-3 means that elements $\operatorname{Dd}(1,1)$, $\operatorname{Dd}(2,2)$ and $\operatorname{Dd}(3,3)$ are chosen; configuration 3-1-2 means that elements $\operatorname{Dd}(1,3), \operatorname{Dd}(2,1)$ and $\operatorname{Dd}(3,2)$ are selected, and so on. Each configuration has a different set of decoupler elements, which is interesting since some choices can result in non-realizable decoupler elements. Thus, the configuration can be selected depending on the realizability, which will be discussed later.

\subsection{Inverted decoupling for $2 \times 2$ processes}

In this section, a detailed study of inverted decoupling for $2 \times 2$ processes is carried out using expression (6). In this case $(n=2)$, there are two possible configurations to choose for the Dd matrix: diagonal elements (configuration 1-2) or off-diagonal elements (configuration 2-1). Hereafter, the Laplace variable $s$ is omitted for simplicity.

\section{a) Configuration 1-2}

Using this configuration, the following expressions (8) for the elements of the inverted decoupling are obtained from (7), which is derived from (6). These are the expressions for general inverted decoupling with configuration 1-2. $\mathrm{q}_{1}$ and $\mathrm{q}_{2}$ are the desired 
equivalent open loop transfer functions to be controlled by the decentralized control, and they can be specified freely as long as the decoupler elements are realizable.

However, in most examples found in the literature on inverted decoupling, two of these elements are set to unity, and so only two elements need to be implemented.

$$
\begin{aligned}
& \left(\begin{array}{cc}
\frac{1}{\operatorname{dd}_{11}} & -\mathrm{do}_{12} \\
-\mathrm{do}_{21} & \frac{1}{\mathrm{dd}_{22}}
\end{array}\right)=\left(\begin{array}{cc}
\frac{g_{11}}{\mathrm{q}_{1}} & \frac{\mathrm{g}_{12}}{\mathrm{q}_{1}} \\
\frac{\mathrm{g}_{21}}{\mathrm{q}_{2}} & \frac{\mathrm{g}_{22}}{\mathrm{q}_{2}}
\end{array}\right) \\
& \mathrm{dd}_{11}=\frac{\mathrm{q}_{1}}{\mathrm{~g}_{11}} \quad \mathrm{do}_{12}=\frac{-\mathrm{g}_{12}}{\mathrm{q}_{1}} \\
& \mathrm{do}_{21}=\frac{-\mathrm{g}_{21}}{\mathrm{q}_{2}} \quad \mathrm{dd}_{22}=\frac{\mathrm{q}_{2}}{\mathrm{~g}_{22}}
\end{aligned}
$$

Usually, the two non-zero elements equal to one are the elements in the direct way, that is, the elements of the Dd matrix. However, this case is only one of the four possible cases according to the two elements chosen to be equal to unity. The control schemes of these different cases are depicted in Figure 4. In an nxn process, the number of possible cases for each configuration is $\mathrm{n}^{\mathrm{n}}$. Nevertheless, the realizability of the decoupler elements in each configuration is always the same, independent of the selected case. For example, as shown in Table 1, for inverted decoupling of TITO processes using configuration 1-2, the pair of decoupler elements that differ from one have the following expressions:

$\frac{-g_{12}}{g_{11}}$ and $\frac{-g_{21}}{g_{22}}$

\begin{tabular}{|c|c|c|}
\hline & Decoupler elements & Decoupled process \\
\hline Case 1 & $\begin{array}{c}\mathrm{dd}_{11}=\mathrm{dd}_{22}=1 \\
\mathrm{do}_{12}=\frac{-\mathrm{g}_{12}}{\mathrm{~g}_{11}}, \quad \mathrm{do}_{21}=\frac{-\mathrm{g}_{21}}{\mathrm{~g}_{22}}\end{array}$ & $\mathrm{Q}=\left(\begin{array}{cc}\mathrm{g}_{11} & 0 \\
0 & \mathrm{~g}_{22}\end{array}\right)$ \\
\hline Case 2 & $\begin{array}{c}\mathrm{dd}_{11}=\mathrm{do}_{21}=1 \\
\mathrm{do}_{12}=\frac{-\mathrm{g}_{12}}{\mathrm{~g}_{11}}, \quad \mathrm{dd}_{22}=\frac{-\mathrm{g}_{21}}{\mathrm{~g}_{22}}\end{array}$ & $\mathrm{Q}=\left(\begin{array}{cc}\mathrm{g}_{11} & 0 \\
0 & -\mathrm{g}_{21}\end{array}\right)$ \\
\hline Case 3 & $\begin{array}{c}\mathrm{do}_{12}=\mathrm{do}_{21}=1 \\
\mathrm{dd}_{11}=\frac{-\mathrm{g}_{12}}{\mathrm{~g}_{11}}, \quad \mathrm{dd}_{22}=\frac{-\mathrm{g}_{21}}{\mathrm{~g}_{22}}\end{array}$ & $\mathrm{Q}=\left(\begin{array}{cc}-\mathrm{g}_{12} & 0 \\
0 & -\mathrm{g}_{21}\end{array}\right)$ \\
\hline Case 4 & $\begin{array}{c}\mathrm{do}_{12}=\mathrm{dd}_{22}=1 \\
\mathrm{dd}_{11}=\frac{-\mathrm{g}_{12}}{\mathrm{~g}_{11}}, \quad \mathrm{do}_{21}=\frac{-\mathrm{g}_{21}}{\mathrm{~g}_{22}}\end{array}$ & $\mathrm{Q}=\left(\begin{array}{cc}-\mathrm{g}_{12} & 0 \\
0 & \mathrm{~g}_{22}\end{array}\right)$ \\
\hline
\end{tabular}

INSERT HERE FIGURE 4

Table 1 - Cases of 2x2 inverted decoupling with two unitary elements (configuration 12)

\section{b) Configuration 2-1}


In this configuration, the general expressions (11) for the inverted decoupling are obtained from (10), which is derived from (6). Note that care should be taken in inverting the Dd matrix.

$$
\begin{aligned}
& \left(\begin{array}{cc}
-\mathrm{do}_{11} & \frac{1}{\mathrm{dd}_{21}} \\
\frac{1}{\mathrm{dd}_{12}} & -\mathrm{do}_{22}
\end{array}\right)=\left(\begin{array}{ll}
\frac{\mathrm{g}_{11}}{\mathrm{q}_{1}} & \frac{\mathrm{g}_{12}}{\mathrm{q}_{1}} \\
\frac{\mathrm{g}_{21}}{\mathrm{q}_{2}} & \frac{\mathrm{g}_{22}}{\mathrm{q}_{2}}
\end{array}\right) \\
& \mathrm{do}_{11}=\frac{-\mathrm{g}_{11}}{\mathrm{q}_{1}} \quad \mathrm{dd}_{12}=\frac{\mathrm{q}_{2}}{\mathrm{~g}_{21}} \\
& \mathrm{dd}_{21}=\frac{\mathrm{q}_{1}}{\mathrm{~g}_{12}} \quad \mathrm{do}_{22}=\frac{-\mathrm{g}_{22}}{\mathrm{q}_{2}}
\end{aligned}
$$

If two of these elements are set to unity, there are again four possible cases (as with configuration 1-2). However, the pair of decoupler elements that differ from one is given by (12), which is the inverse of (9) in the other configuration.

$\frac{-g_{11}}{g_{12}}$ and $\frac{-g_{22}}{g_{21}}$

The cases in which the elements of the Dd matrix are equal to unity (case 1 in configuration 1-2, for instance) are the only ones found in the literature.

\subsection{Inverted decoupling for $3 \times 3$ processes}

For $3 \times 3$ processes, the procedure is the same: we obtain the expressions from (6) after choosing the configuration. In this case, there are six (3!) possible configurations according to the three elements of Dd chosen to be non-zero. In the following, two examples of these configurations are shown. Using configuration 1-2-3 (diagonal elements of Dd) in (6), equation (13) is obtained. From this, the expressions of the decoupler elements (14) are easily derived.

$$
\begin{aligned}
& \left(\begin{array}{ccc}
\frac{1}{\mathrm{dd}_{11}} & -\mathrm{do}_{12} & -\mathrm{do}_{13} \\
-\mathrm{do}_{21} & \frac{1}{\mathrm{dd}_{22}} & -\mathrm{do}_{23} \\
-\mathrm{do}_{31} & -\mathrm{do}_{32} & \frac{1}{\mathrm{dd}_{33}}
\end{array}\right)=\left(\begin{array}{ccc}
\frac{g_{11}}{\mathrm{q}_{1}} & \frac{\mathrm{g}_{12}}{\mathrm{q}_{1}} & \frac{\mathrm{g}_{13}}{\mathrm{q}_{1}} \\
\frac{\mathrm{g}_{21}}{\mathrm{q}_{2}} & \frac{\mathrm{g}_{22}}{\mathrm{q}_{2}} & \frac{\mathrm{g}_{23}}{\mathrm{q}_{2}} \\
\frac{\mathrm{g}_{31}}{\mathrm{q}_{3}} & \frac{\mathrm{g}_{32}}{\mathrm{q}_{3}} & \frac{\mathrm{g}_{33}}{\mathrm{q}_{3}}
\end{array}\right) \\
& \mathrm{dd}_{11}=\frac{\mathrm{q}_{1}}{\mathrm{~g}_{11}} \quad \mathrm{do}_{12}=\frac{-\mathrm{g}_{12}}{\mathrm{q}_{1}} \quad \mathrm{do}_{13}=\frac{-\mathrm{g}_{13}}{\mathrm{q}_{1}} \\
& \mathrm{do}_{21}=\frac{-\mathrm{g}_{21}}{\mathrm{q}_{2}} \quad \mathrm{dd}_{22}=\frac{\mathrm{q}_{2}}{\mathrm{~g}_{22}} \quad \mathrm{do}_{23}=\frac{-\mathrm{g}_{23}}{\mathrm{q}_{2}} \\
& \mathrm{do}_{31}=\frac{-\mathrm{g}_{31}}{\mathrm{q}_{3}} \quad \mathrm{do}_{32}=\frac{-\mathrm{g}_{32}}{\mathrm{q}_{3}} \quad \mathrm{dd}_{33}=\frac{\mathrm{q}_{3}}{\mathrm{~g}_{33}}
\end{aligned}
$$


Using configuration 2-1-3 (15) and operating in the same way, the corresponding expressions of the decoupler elements (16) are achieved.

$$
\begin{aligned}
& \left(\begin{array}{ccc}
-\mathrm{do}_{11} & \frac{1}{\mathrm{dd}_{21}} & -\mathrm{do}_{13} \\
\frac{1}{\mathrm{dd}_{12}} & -\mathrm{do}_{22} & -\mathrm{do}_{23} \\
-\mathrm{do}_{31} & -\mathrm{do}_{32} & \frac{1}{\mathrm{dd}_{33}}
\end{array}\right)=\left(\begin{array}{ccc}
\frac{g_{11}}{\mathrm{q}_{1}} & \frac{\mathrm{g}_{12}}{\mathrm{q}_{1}} & \frac{\mathrm{g}_{13}}{\mathrm{q}_{1}} \\
\frac{\mathrm{g}_{21}}{\mathrm{q}_{2}} & \frac{\mathrm{g}_{22}}{\mathrm{q}_{2}} & \frac{\mathrm{g}_{23}}{\mathrm{q}_{2}} \\
\frac{\mathrm{g}_{31}}{\mathrm{q}_{3}} & \frac{\mathrm{g}_{32}}{\mathrm{q}_{3}} & \frac{\mathrm{g}_{33}}{\mathrm{q}_{3}}
\end{array}\right) \\
& \mathrm{do}_{11}=\frac{-\mathrm{g}_{11}}{\mathrm{q}_{1}} \quad \mathrm{dd}_{12}=\frac{\mathrm{q}_{2}}{\mathrm{~g}_{21}} \quad \mathrm{do}_{13}=\frac{-\mathrm{g}_{13}}{\mathrm{q}_{1}} \\
& \mathrm{dd}_{21}=\frac{\mathrm{q}_{1}}{\mathrm{~g}_{12}} \quad \mathrm{do}_{22}=\frac{-\mathrm{g}_{22}}{\mathrm{q}_{2}} \quad \mathrm{do}_{23}=\frac{-\mathrm{g}_{23}}{\mathrm{q}_{2}} \\
& \mathrm{do}_{31}=\frac{-\mathrm{g}_{31}}{\mathrm{q}_{3}} \quad \mathrm{do}_{32}=\frac{-\mathrm{g}_{32}}{\mathrm{q}_{3}} \quad \mathrm{dd}_{33}=\frac{\mathrm{q}_{3}}{\mathrm{~g}_{33}}
\end{aligned}
$$

These expressions are as simple as in $2 \mathrm{x} 2$ processes. If three elements are fixed to unity, there are 27 cases for each configuration. Nevertheless, we study only the case in which the elements of the Dd matrix are equal to unity. In this case and using configuration 12-3, the following expressions are obtained:

$$
\begin{array}{lll}
\mathrm{dd}_{11}=1 & \mathrm{do}_{12}=\frac{-\mathrm{g}_{12}}{\mathrm{~g}_{11}} & \mathrm{do}_{13}=\frac{-\mathrm{g}_{13}}{\mathrm{~g}_{11}} \\
\mathrm{do}_{21}=\frac{-\mathrm{g}_{21}}{\mathrm{~g}_{22}} & \mathrm{dd}_{22}=1 & \mathrm{do}_{23}=\frac{-\mathrm{g}_{23}}{\mathrm{~g}_{22}} \\
\mathrm{do}_{31}=\frac{-\mathrm{g}_{31}}{\mathrm{~g}_{33}} & \mathrm{do}_{32}=\frac{-\mathrm{g}_{32}}{\mathrm{~g}_{33}} & \mathrm{dd}_{33}=1
\end{array}
$$

The apparent processes are the diagonal elements of G(s), as obtained with ideal decoupling, and the decoupler elements are as simple as the simplified decoupler elements for $2 \mathrm{x} 2$ processes.

In [24], an interaction compensator is designed for a 3x3 process as a static compensator. This design is carried out as in the simplified decoupling case. The expressions of simplified decoupling for $3 \times 3$ processes are shown in (18) where $G^{\mathrm{ij}}$ is the cofactor corresponding to $g_{i j}(s)$ of $G(s)$. Then, the realization is made as with the inverse decoupling structure using the static simplified decoupling elements for $3 \mathrm{x} 3$ processes (18). It is said that the resulting interaction compensator obtains the apparent process of the ideal decoupling case in steady-state conditions. This last statement is wrong, because it would only be correct for TITO processes, not for systems with greater dimensions.

In the case of $3 \times 3$ processes, the cofactor of $g_{11}$ is $G^{11}=g_{22} \cdot g_{33}-g_{32} \cdot g_{23}$. Therefore, it is easy to find that the expressions of the inverted decoupler elements in (17) are much simpler than the simplified decoupler elements in (18). In addition, the apparent processes are given by equation (3), which is much more complex than the diagonal elements of $\mathrm{G}(\mathrm{s})$. 


$$
D=\left(\begin{array}{ccc}
1 & \frac{G^{21}}{G^{22}} & \frac{G^{31}}{G^{33}} \\
\frac{G^{12}}{G^{11}} & 1 & \frac{G^{32}}{G^{33}} \\
\frac{G^{13}}{G^{11}} & \frac{G^{23}}{G^{22}} & 1
\end{array}\right)
$$

\subsection{General expressions of inverted decoupling}

From (6) and the equations of the previous sections, it is possible to obtain the general expressions of inverted decoupling for $n x n$ processes. If the configuration $\left\{\mathrm{p}_{1}-\mathrm{p}_{2}-\ldots-\mathrm{p}_{\mathrm{i}}-\right.$ $\left.\ldots \mathrm{p}_{\mathrm{n}-1}-\mathrm{p}_{\mathrm{n}}\right\}$ is chosen, the elements that differ from zero of the Dd and Do matrices are given by (19) and (20), respectively. The transfer functions of the apparent process $q_{i}(s)$ can be selected in any way that assures the realizability of these decoupler elements.

$$
\begin{aligned}
& d d_{i j}=\frac{q_{j}}{g_{j i}} \quad \forall i ; \quad j=p_{i} \\
& d o_{i j}=\frac{-g_{i j}}{q_{i}} \quad \forall i, j ; \quad / \quad i \neq p_{j}
\end{aligned}
$$

The following advantages of inverted decoupling are concluded from these general expressions:

- The apparent processes do not contain sums of transfer functions, and therefore, tuning of the diagonal controllers is easier. In multivariable processes with strong cross couplings, even if the elements of the system have simple dynamics, conventional decoupling may result in complicated diagonal apparent processes consisting of parallel coupled processes that may have different signs and different time delays [12]. An example of such a diagonal element is:

$$
G(s)=\frac{1.2}{(0.5 s+1)(0.7 s+1)} e^{-4 s}-\frac{1}{(3 s+1)(2 s+1)} e^{-s}
$$

which consists of a difference between two fairly simple transfer functions. The step response of this process is shown in Figure 5. If PID controllers are used to control a system with diagonal elements like (21), design methods that rely on simple process dynamics, like step response methods [25], are not appropriate.

\section{INSERT HERE FIGURE 5}

- Decoupler elements do not contain either sum of transfer functions, so they are easy to design. Using conventional decoupling, in some cases, it is possible to have decoupler

elements with complexity similar to that of process (21), and therefore, they are difficult to implement. 
- The complexity of the decoupler elements and apparent processes is always the same, independent of the size of the process. With conventional decoupling, these elements become more complex as the system size increases.

Nevertheless, inverted decoupling presents an important disadvantage: due to stability problems, it cannot be applied to processes with RHP zeros in the determinant of the transfer matrix G(s). For internal stability, these RHP zeros should appear in the apparent process. In a conventional decoupling structure, RHP zeros of the determinant of $\mathrm{G}(\mathrm{s})$ can be included in the desired equivalent open loop transfer functions $\mathrm{q}_{i}(\mathrm{~s})$. However, this is not possible using inverted decoupling, because such RHP zeros would appear as unstable poles in some decoupler elements $\mathrm{do}_{\mathrm{ij}}(\mathrm{s})$. If the multivariable RHP zero is associated with a single output and is therefore included in the process transfer functions of the same row, inverted decoupling can be applied because the RHP zero will be cancelled.

Therefore, as mentioned in [23], it seems that inverted decoupling cannot be applied in general to a process with a determinant that has RHP zeros. To apparently solve it, in [23], the decoupler structure is changed from inverted decoupling to conventional ideal decoupling without feedback, and so, the inverted decoupling structure is not used.

When the non-zero elements of the Dd matrix in (19) are fixed to unity, the expressions of the decoupler elements and apparent processes $q_{i}(s)$ are given by (22), (23) and (24).

$$
\begin{aligned}
& d d_{i j}=1 \quad \forall i ; \quad j=p_{i} \\
& d o_{i j}=\frac{-g_{i j}}{g_{i k}} \quad \forall i, j ; / i \neq p_{j} ; k / p_{k}=i \\
& q_{j}=g_{j i} \forall j ; i / p_{i}=j
\end{aligned}
$$

In this case (non-zero $\mathrm{dd}_{\mathrm{ij}}=1$ ), inverted decoupling presents several practical advantages over conventional decoupling, which makes this structure very interesting from a practical point of view. These advantages [11] are the following:

- The apparent process seen by each controller is the same as that obtained if there was no decoupling and the alternate controllers were in the manual mode.

- Inverted decoupling can often be implemented within a DCS using PID function blocks with feedforward inputs. This will automatically provide such features as initialization and bumpless transfer between manual and automatic.

- The antireset windup feature of the PID, combined with its feedforward input, can be used to directly take into account the saturation of the manipulated variables when inverted decoupling is implemented.

- When decoupler outputs are used as cascade setpoints to lower level controllers, each decoupled control loop is immune to abnormalities (e.g., a valve at a limit or a secondary controller in manual) in the secondary of the opposite control loops.

Conventional decoupler networks present several practical disadvantages: problems like bumpless transfer or antireset windup are more difficult to solve, and the controller parameters should change when some decoupler element is disenable, since the apparent process will be different. In addition, for high dimensional systems ( $3 \times 3$ or $4 \times 4)$ the 
design of conventional decouplers becomes more complicated and important approximations are usually necessary. For these reasons, many industrial multivariable processes are preferred to be controlled by decentralized PID controllers due to the easiness of implementation and tuning.

Nevertheless, using inverted decoupling (with non-zero $\mathrm{dd}_{\mathrm{ij}}=1$ ), as the apparent process is the same for such processes, their performance could be easily improved simply adding decoupling blocks (lead-lags plus time delay blocks). This is important, because it means that inverted decoupling can be used in most modern DCS systems using the standard blocks that are already available in their function block library. Thanks to the easiness of implementation and achieving bumpless transfer, it would be easy to alternate between a decentralized or centralized control without modifying the PID parameters. For this reason, inverted decoupling may be a good and easy way to improve the performance of industrial multivariable processes with interaction problems.

\section{Decoupler realizability}

The realizability requirement for the decoupler is that all of its elements must be proper, causal and stable. For processes with time delays or non-minimum-phase zeros, direct calculation of the decoupler can lead to elements with prediction or right-half-plane (RHP) poles. This problem is discussed in [23] for inverted decoupling of 2x2 processes.

In this section, the conditions that a specified configuration needs to satisfy in order to be realizable are discussed. In addition, the constraints on the apparent process are indicated. There are three aspects to take into account and to be inspected for each row:

- Non-causal time delays $\theta_{i j}$ must be avoided in decoupler elements. If $\mathrm{g}_{\mathrm{ik}}$ is the transfer function of the row $i$ with the smallest time delay $\theta_{i k}$, the element $\mathrm{dd}_{\mathrm{ki}}$ of Dd should be different from zero. In addition, the time delay of the $q_{i}$ apparent process $\left(\theta_{q_{i}}\right)$ must fulfill (25):

$$
\theta_{i k} \leq \theta_{q_{i}} \leq \min _{j \neq k}\left(\theta_{i j}\right)
$$

- Decoupler elements must be proper, that is, the relative degrees $r_{i j}$ must be greater than or equal than zero. Similar to the case of time delays, if $g_{i k}$ is the transfer function of the row $i$ with the smallest relative degree $r_{i k}$, the element $\mathrm{dd}_{\mathrm{ki}}$ of Dd should be different from zero. In addition, the relative degree of the $\mathrm{q}_{\mathrm{i}}$ apparent process $\left(\mathrm{r}_{\mathrm{qi}}\right)$ must fulfill (26):

$$
r_{i k} \leq r_{q_{i}} \leq \min _{j \neq k}\left(r_{i j}\right)
$$

- When some transfer function $\mathrm{g}_{\mathrm{im}}$ has a RHP zero, the element $\mathrm{dd}_{\mathrm{mi}}$ of Dd should not be different from zero in order to avoid this zero becoming a RHP pole in some decoupler element. When the same zero appears in all elements of the same row, it is necessary to check the multiplicity $\eta_{i j}$ in each element. Again, similar to the other cases, if $g_{i k}$ is the transfer function of the row $i$ with the smallest RHP zero multiplicity $\eta_{i k}$, the 
element $\mathrm{dd}_{\mathrm{ki}}$ should be non-zero. This RHP zero must appear in the $\mathrm{q}_{\mathrm{i}}$ apparent process with a multiplicity $\left(\eta_{q_{i}}\right)$ that fulfils:

$\eta_{i k} \leq \eta_{q_{i}} \leq \min _{j \neq k}\left(\eta_{i j}\right)$

From (25), (26) and (27), it is found that when the smallest value (time delay, relative degree or RHP zero multiplicity) is shared by two or more transfer functions of the same row, there are more possibilities for the configuration, but the flexibility (time delay or relative degree) of the apparent process $q_{i}$ is limited to this common smallest value of the row.

When two or more elements of Dd must be selected in the same column to satisfy the previous conditions in all rows, there are no realizable configurations. Then, it is necessary to insert an additional diagonal block N(s) between the system $\mathrm{G}(\mathrm{s})$ and the inverted decoupler $\mathrm{D}(\mathrm{s})$ in order to modify the process and to force the non-realizable elements into realizability. Then, inverted decoupling can be applied to the new process $\mathrm{G}^{\mathrm{N}}(\mathrm{s})=\mathrm{G}(\mathrm{s}) \cdot \mathrm{N}(\mathrm{s})$.

$\mathrm{N}(\mathrm{s})$ is a diagonal block with the necessary extra dynamics. If there are no realizability problems in row $i$, the $\mathrm{N}(\mathrm{i}, \mathrm{i})$ element is equal to the unity. If the non-realizability comes from an element with a non-causal time delay, an additional time delay $\left(e^{-\theta_{i} s}\right)$ is inserted in the corresponding diagonal element of $\mathrm{N}(\mathrm{s})$ to obtain realizability. If it comes from a RHP zero $\mathrm{z}$, which has become an unstable pole, the following element is used in $\mathrm{N}(\mathrm{s})$ :

$\left(\frac{-s+z}{s+z^{*}}\right)^{\eta_{i}}$

where $\mathrm{z}^{*}$ is the complex conjugate of $\mathrm{z}$. If it comes from a properness problem, a simple stable pole with the adequate multiplicity can be inserted as follows:

$\frac{1}{(\lambda s+1)^{r_{i}}}$

For illustration, considering the following example [5]

$\mathrm{G}(\mathrm{s})=\left(\begin{array}{cc}\frac{e^{-2 s}}{s+2} & \frac{-e^{-6 s}}{s+2} \\ \frac{(s-0.5) \cdot e^{-3 s}}{(s+2)^{2}} & \frac{(s-0.5)^{2} \cdot e^{-8 s}}{2(s+2)^{3}}\end{array}\right)$

This process has a multivariable RHP zero at $s=0.5$. Nevertheless, it is associated with a single output, the second one, and therefore, inverted decoupling can be applied.

However, this RHP zero appears in the two process transfer functions of the second row with different multiplicity. According to the previous RHP zero condition, the element $\mathrm{dd}_{21}$ should be selected to be non-zero in the Dd matrix because element $\mathrm{G}(2,1)$ has the smallest RHP zero multiplicity. In addition, it has the smallest time delay of the second 
row. In the first row, due to time delay condition, the element $\mathrm{dd}_{11}$ should be selected to be non-zero in the Dd matrix. Since elements $\mathrm{dd}_{11}$ and $\mathrm{dd}_{21}$ are in the same column, no configuration is initially realizable. To achieve realizability, an extra time delay of 4 units has to be added in the first input. In this case, the new process to be decoupled is given by (31), and using configuration $2-1$, the element $\mathrm{dd}_{12}$ can be selected in the first row. Then, according to (11), the decoupler matrices are given by (32) and the apparent decoupled process is composed by off diagonal elements of (31). The RHP zero appears in the apparent process of the second output, which is necessary for internal stability.

$$
\mathrm{G}^{\mathrm{N}}(\mathrm{s})=\left(\begin{array}{cc}
\frac{e^{-6 s}}{s+2} & \frac{-e^{-6 s}}{s+2} \\
\frac{(s-0.5) \cdot e^{-7 s}}{(s+2)^{2}} & \frac{(s-0.5)^{2} \cdot e^{-8 s}}{2(s+2)^{3}}
\end{array}\right)
$$

$$
\mathrm{Dd}=\left(\begin{array}{ll}
0 & 1 \\
1 & 0
\end{array}\right) \quad \mathrm{Do}=\left(\begin{array}{cc}
1 & 0 \\
0 & \frac{-0.5(s-0.5) \cdot e^{-s}}{(s+2)}
\end{array}\right)
$$

Generally, it is preferable to add the minimum extra dynamics. Therefore, after checking the necessary additional dynamics of each configuration, we choose the one with fewer RHP zeros or time delays in N(s).

The problem of determining the minimal N(s) for a given configuration can be formulated as a linear programming problem. For instance, the next example of a 3x3 process (33) shows the procedure when it is only necessary to add extra time delays in $\mathrm{N}(\mathrm{s})$. This process, the Tyreus distillation column [26], does not have any configurations in which all inverted decoupling elements are realizable. According to the previous condition, the elements $\mathrm{dd}_{11}, \mathrm{dd}_{23}$ and $\mathrm{dd}_{33}$ should be selected to be nonzero in the Dd matrix because they have the smallest time delays by row. However, two of them are in the same column, so no configuration is initially realizable.

$$
\mathrm{G}_{\mathrm{T}}(\mathrm{s})=\left(\begin{array}{ccc}
\frac{1.986 e^{-0.71 s}}{66.7 s+1} & \frac{-5.24 e^{-60 s}}{400 s+1} & \frac{-5.984 e^{-2.24 s}}{14.29 s+1} \\
\frac{-0.0204 e^{-0.59 s}}{(7.14 s+1)^{2}} & \frac{0.33 e^{-0.68 s}}{(2.38 s+1)^{2}} & \frac{-2.38 e^{-0.42 s}}{(1.43 s+1)^{2}} \\
\frac{-0.374 e^{-7.75 s}}{22.22 s+1} & \frac{11.3 e^{-3.79 s}}{(21.74 s+1)^{2}} & \frac{9.811 e^{-1.59 s}}{11.36 s+1}
\end{array}\right)
$$

To force realizability, the diagonal block of extra time delays $\mathrm{N}(\mathrm{s})=\operatorname{diag}\left(e^{-n_{1} s}, e^{-n_{2} s}, e^{-n_{3} s}\right)$ is proposed, and the goal is to specify the smallest $\mathrm{n}_{\mathrm{i}} \geq 0$ that obtains realizability for the inverted decoupling. The time delay matrix $\Theta$ of the new process $\mathrm{G}(\mathrm{s}) \cdot \mathrm{N}(\mathrm{s})$ is given by (34). 
$\Theta=\left(\begin{array}{ccc}0.71+n_{1} & 60+n_{2} & 2.24+n_{3} \\ 0.59+n_{1} & 0.68+n_{2} & 0.42+n_{3} \\ 7.75+n_{1} & 3.79+n_{2} & 1.59+n_{3}\end{array}\right)$

Configuration 1-2-3 is selected, and consequently, the diagonal elements of (34) should be the smallest. According to (17), where the same configuration is used, the time delay matrix of the Do matrix is given by (35), and its time delays must be equal to or greater than zero in order to obtain realizability in the decoupler $\left(\Theta_{D o} \geq 0\right)$.

$\Theta_{D o}=\left(\begin{array}{ccc}0 & 59.29+n_{2}-n_{1} & 1.53+n_{3}-n_{1} \\ -0.09+n_{1}-n_{2} & 0 & -0.26+n_{3}-n_{2} \\ 6.16+n_{1}-n_{3} & 2.2+n_{2}-n_{3} & 0\end{array}\right)$

This condition can be represented as follows:

$$
A \cdot \Theta_{N} \geq b \Leftrightarrow\left(\begin{array}{rrr}
-1 & 1 & 0 \\
-1 & 0 & 1 \\
1 & -1 & 0 \\
0 & -1 & 1 \\
1 & 0 & -1 \\
0 & 1 & -1
\end{array}\right) \cdot\left(\begin{array}{c}
n_{1} \\
n_{2} \\
n_{3}
\end{array}\right) \geq\left(\begin{array}{c}
-59.29 \\
-1.53 \\
0.09 \\
0.26 \\
-6.16 \\
-2.2
\end{array}\right)
$$

Then, the problem can be formulated as the following linear programming minimization (37), which obtains the smallest sum of time delays.

$$
\begin{aligned}
& \min \sum n_{i} \\
& \text { subject to : }-A \cdot \Theta_{N} \leq-b, \quad n_{i} \geq 0
\end{aligned}
$$

For the example under study, the solution is $\mathrm{n}_{1}=0.09, \mathrm{n}_{2}=0$ and $\mathrm{n}_{3}=0.26$, and configuration 1-2-3 is the only one that achieves realizability. Using other possible configurations, there is no feasible region in problem (37), independent of $\mathrm{N}(\mathrm{s})$.

This procedure can be similarly applied to realizability problems that arise from relative degrees or RHP zeros. When the inclusion of an additional block N(s) is necessary to obtain realizability, one of the main inconveniences is a loss of the practical advantages of the inverted decoupling, as mentioned in [11].

\section{Examples}

In this section, the proposed methodology is applied to three simulated processes of different dimensions. Additionally, its effectiveness is verified in a real quadruple tank plant.

\subsection{Example 2x2: An industrial-scale polymerization reactor}

This process [7] is an industrial-scale polymerization reactor given by (38), where the time scales are in hours. The two controlled variables are measurements of the reactor 
condition, and the two manipulated variables are the setpoints of the two reactor feed flow loops.

$$
\mathrm{G}_{\mathrm{R}}(\mathrm{s})=\left(\begin{array}{cc}
\frac{22.89 \cdot e^{-0.2 s}}{4.572 s+1} & \frac{-11.64 \cdot e^{-0.4 s}}{1.807 s+1} \\
\frac{4.689 \cdot e^{-0.2 s}}{2.174 s+1} & \frac{5.80 \cdot e^{-0.4 s}}{1.801 s+1}
\end{array}\right)
$$

In this case, due to the time delays of the system, there are no realizable configurations for inverted decoupling. To obtain realizability, it is necessary to add an extra time delay associated with the first input. The diagonal block $N(s)$ is given by $n_{11}(s)=e^{-0.2 s}$ and $\mathrm{n}_{22}(\mathrm{~s})=1$. Then, the new apparent process is the following:

$$
\mathrm{G}_{\mathrm{R}}^{\mathrm{N}}(\mathrm{s})=\left(\begin{array}{cc}
\frac{22.89 \cdot e^{-0.4 s}}{4.572 s+1} & \frac{-11.64 \cdot e^{-0.4 s}}{1.807 s+1} \\
\frac{4.689 \cdot e^{-0.4 s}}{2.174 s+1} & \frac{5.80 \cdot e^{-0.4 s}}{1.801 s+1}
\end{array}\right)
$$

As there are no RHP zeros in the determinant of G(s), inverted decoupling is applicable. If configuration 1-2 is chosen and the two elements of the Dd(s) matrix are fixed to one, according to Table 1 , the apparent processes $\mathrm{q}_{1}(\mathrm{~s})$ and $\mathrm{q}_{2}(\mathrm{~s})$ are given by $\mathrm{g}_{11}(\mathrm{~s})$ and $\mathrm{g}_{22}(\mathrm{~s})$ of (39). The other two decoupler elements of Do(s) are collected in Table 2.

After determining the decoupler network, the parameters of the decentralized controller can be tuned independently for the corresponding $\mathrm{q}_{\mathrm{i}}(\mathrm{s})$. Therefore, the existing SISO PID tuning methods can be directly applied to guarantee the stability and performance of each loop. In this work, gain margin specifications are used to tune the PID controllers according to the tuning rules in [27]. A gain margin of 5 is specified for both loops. The parameters of the two resulting PI controllers are shown in Table 2.

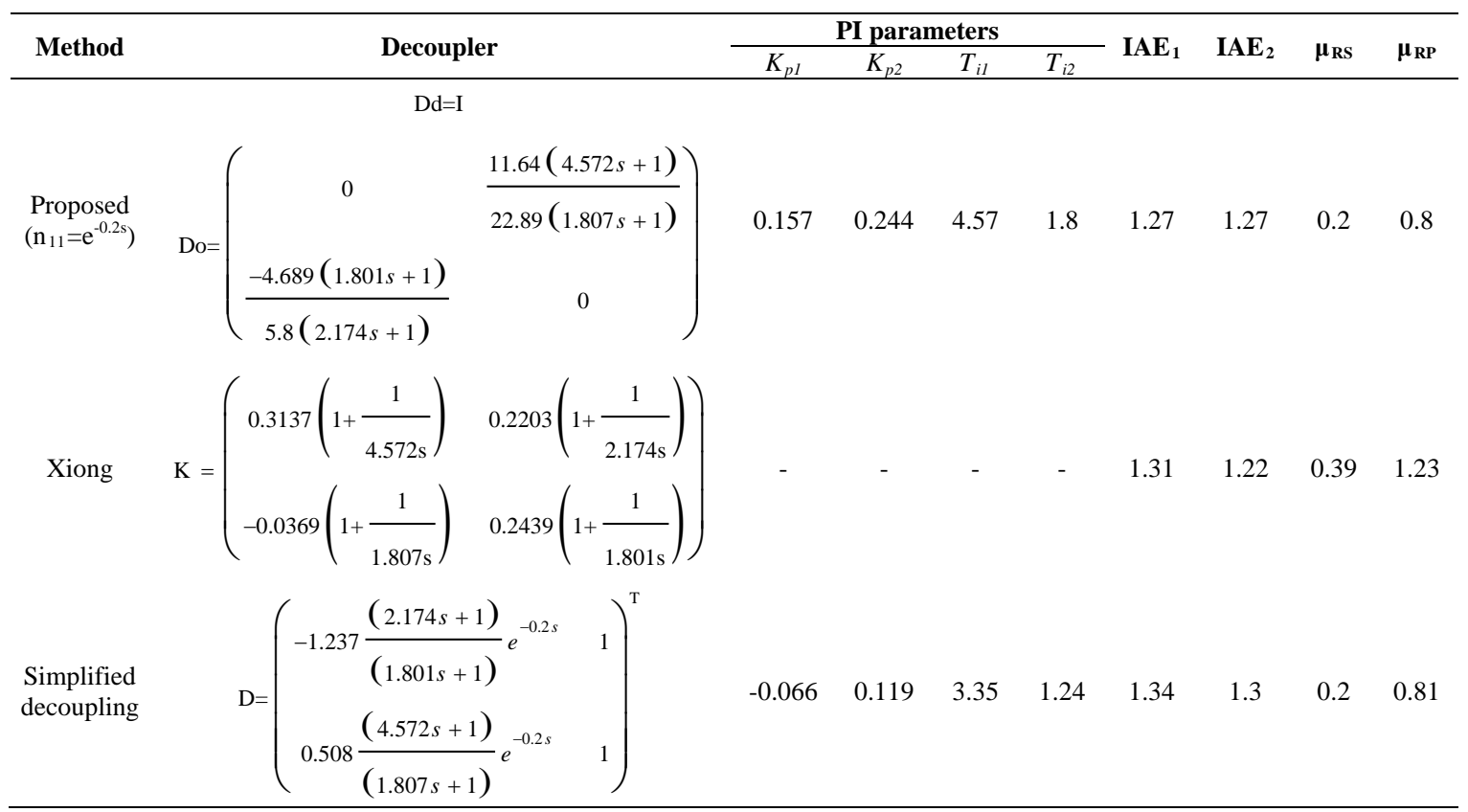




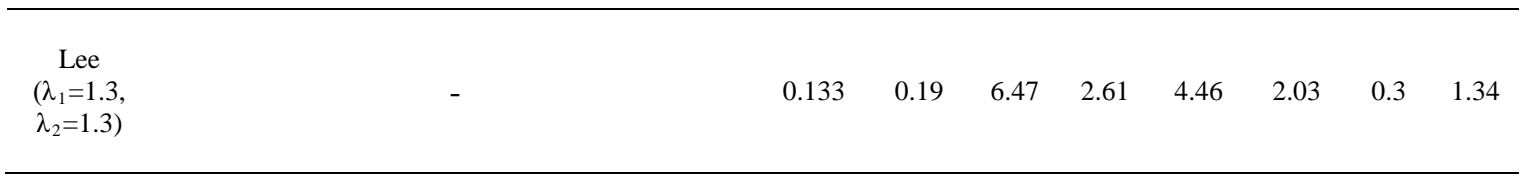

Table 2 - Different control methods for Example 1 with performance and robustness indices

Next, in order to verify the nominal control system performance, the closed loop system responses are shown in Figure 6. There are unit step changes at $t=1 \mathrm{~h}$, in the first reference, and at $\mathrm{t}=25 \mathrm{~h}$, in the second one. The IAE of each loop is obtained as performance index and collected in Table 2. For comparison, other control methodologies are also shown in the figure: the centralized PID control of Xiong [7], a multiloop PI controller based on Lee's method [28], and a simplified decoupling using the third configuration in [10]. They are tuned trying to achieve similar performance (a gain margin of 5 in each loop). All parameters of these other controllers are shown in Table 2 with the corresponding IAE indices. With regard to simplified decoupling, it is interesting to note the greater complexity of the apparent decoupled processes (40).

$$
\begin{aligned}
& \mathrm{q}_{\text {simp1 }}(\mathrm{s})=\frac{-\left(13.92 s^{2}+12.56 s+2.685\right) \cdot e^{-0.4 s}}{s^{3}+1.327 s^{2}+0.5498 s+0.06721} \\
& \mathrm{q}_{\text {simp2 }}(\mathrm{s})=\frac{\left(5.996 s^{2}+5.411 s+1.157\right) \cdot e^{-0.4 s}}{s^{3}+1.569 s^{2}+0.8172 s+0.1413}
\end{aligned}
$$

\section{INSERT HERE FIGURE 6}

The proposed design presents better performance. In the outputs, the inverted decoupling is better in terms of overshoot, decoupling and settling time. It also has the best average IAE index. The control signals are less aggressive, and their peaks are smaller. The response of simplified decoupling is quite similar.

In order to evaluate the robustness of the controllers, a $\mu$-analysis is carried out in presence of diagonal multiplicative input uncertainty. Multiplicative input uncertainty is represented as illustrated in Figure 7, where $\Delta_{\mathrm{I}}(\mathrm{s})$ is the disturbance and $\mathrm{W}_{\mathrm{I}}(\mathrm{s})$ and $\mathrm{W}_{\mathrm{P}}(\mathrm{s})$ are the diagonal weights for uncertainty and performance, respectively. In this example, the selected weights are

$$
\begin{aligned}
& \mathrm{W}_{\mathrm{I}}(\mathrm{s})=\mathrm{W}_{\mathrm{I}}(\mathrm{s}) \cdot \mathrm{I}=\frac{(0.1 \mathrm{~s}+0.2)}{0.05 \mathrm{~s}+1} \cdot \mathrm{I} \\
& \mathrm{W}_{\mathrm{P}}(\mathrm{s})=\mathrm{W}_{\mathrm{P}}(\mathrm{s}) \cdot \mathrm{I}=\frac{(\mathrm{s} / 2.2+0.3)}{\mathrm{s}} \cdot \mathrm{I}
\end{aligned}
$$

The weight $\mathrm{w}_{\mathrm{I}}(\mathrm{s})$ can be loosely interpreted as the process inputs increase by up to $200 \%$ uncertainty at high frequencies and by almost $20 \%$ uncertainty in the low frequency range. The performance weight $\mathrm{w}_{\mathrm{P}}(\mathrm{s})$ specifies integral action, a maximum peak for $\bar{\sigma}(S)$ of $\mathrm{M}_{\mathrm{s}}=2.2$ and a bandwidth of about $0.3 \mathrm{rad} / \mathrm{s}$.

\section{INSERT HERE FIGURE 7}


To achieve robust stability the necessary and sufficient condition [16] is

$$
\mu_{\mathrm{RS}}=\mu\left[-\mathrm{W}_{\mathrm{I}}(\mathrm{s}) \mathrm{T}_{\mathrm{I}}(\mathrm{s})\right]<1 \quad \forall \omega
$$

where $\mu$ is the structured singular value (SSV) and $\mathrm{T}_{\mathrm{I}}(\mathrm{s})=\mathrm{K}(\mathrm{s}) \mathrm{G}(\mathrm{s})(\mathrm{I}+\mathrm{K}(\mathrm{s}) \mathrm{G}(\mathrm{s}))^{-1}$ is the input complementary sensitivity function. To evaluate if the closed loop system will respect the desired performance even in presence of diagonal multiplicative input uncertainty, the necessary and sufficient condition [16] is

$$
\mu_{\mathrm{RP}}=\mu\left[\begin{array}{cc}
-\mathrm{W}_{\mathrm{I}}(\mathrm{s}) \mathrm{T}_{\mathrm{I}}(\mathrm{s}) & -\mathrm{W}_{\mathrm{I}}(\mathrm{s}) \mathrm{K}(\mathrm{s}) \mathrm{S}(\mathrm{s}) \\
\mathrm{W}_{\mathrm{P}}(\mathrm{s}) \mathrm{S}(\mathrm{s}) \mathrm{G}(\mathrm{s}) & \mathrm{W}_{\mathrm{P}}(\mathrm{s}) \mathrm{S}(\mathrm{s})
\end{array}\right]<1 \quad \forall \omega
$$

where $S(s)=(I+G(s) K(s))^{-1}$ is the sensitivity function and $T(s)=G(s) K(s)(I+G(s) K(s))^{-1}$ is the complementary sensitivity function.

Figure 8 shows the SSV for robust stability and robust performance for the different controllers. The robust stability (RS) is clearly smaller than one for all frequencies, indicating that the systems will remain stable in spite of an uncertainty of $20 \%$ on each process input. The peak values are shown in Table 2 . The proposed method and the simplified decoupling have the smallest values. The robust performance analysis shows that the proposed method and simplified decoupling satisfy the robust performance (RP) condition (43). For the other controllers, the performance will deteriorate at frequency range of [1-10] rad/s, where the peaks appear. These values are also collected in Table 2. The multiloop PI controller of Lee has a good robust stability; however, it shows the worst robust performance, even at low frequencies, due to the strong interactions in the first loop.

\section{INSERT HERE FIGURE 8}

\subsection{Example 3x3: Tyreus distillation column}

This process [26] is a sidestream column separating a ternary mixture, where a feed containing 10 percent benzene, 45 percent toluene and 45 percent o-xylene is separated in a single column into three product streams. The transfer function matrix is given by (33), where the controlled and manipulated variables are $\mathrm{y}_{1}$ (toluene impurity in the distillate); $\mathrm{y}_{2}$ (benzene impurity in the sidestream); $\mathrm{y}_{3}$ (toluene impurity in the bottom); $\mathrm{u}_{1}$ (reflux ratio); $\mathrm{u}_{2}$ (sidestream flow rate); $\mathrm{u}_{3}$ (reboil duty).

As was explained in Section 3, there are no realizable configurations for this process due to the time delays. Thus, it is necessary to include an additional block N(s) with delays. To achieve realizability by adding the minimum quantity of delays, the best choice is configuration 1-2-3 with $\mathrm{n}_{11}(\mathrm{~s})=\mathrm{e}^{-0.09 \mathrm{~s}}, \mathrm{n}_{22}(\mathrm{~s})=1$ and $\mathrm{n}_{33}(\mathrm{~s})=\mathrm{e}^{-0.26 \mathrm{~s}}$. The new apparent process to design the inverted decoupling is: 


$$
\mathrm{G}_{\mathrm{T}}^{\mathrm{N}}(\mathrm{s})=\left(\begin{array}{cccc}
\frac{1.986 e^{-0.8 s}}{66.7 s+1} & \frac{-5.24 e^{-60 s}}{400 s+1} & \frac{-5.984 e^{-2.5 s}}{14.29 s+1} \\
\frac{-0.0204 e^{-0.68 s}}{(7.14 s+1)^{2}} & \frac{0.33 e^{-0.68 s}}{(2.38 s+1)^{2}} & \frac{-2.38 e^{-0.68 s}}{(1.43 s+1)^{2}} \\
\frac{-0.374 e^{-7.84 s}}{22.22 s+1} & \frac{11.3 e^{-3.79 s}}{(21.74 s+1)^{2}} & \frac{9.811 e^{-1.85 s}}{11.36 s+1}
\end{array}\right)
$$

Therefore, using configuration 1-2-3 and fixing the three elements of the $\operatorname{Dd}(\mathrm{s})$ matrix to one, the open loop processes are the diagonal elements of (44), and the decoupler matrix Do(s) can be calculated according to (17), obtaining:

$$
\operatorname{Do}(\mathrm{s})=\left(\begin{array}{ccc}
0 & \frac{2.638(66.7 s+1) e^{-59.2 s}}{(400 s+1)} & \frac{3.013(66.7 s+1) e^{-1.7 s}}{(14.29 s+1)} \\
\frac{0.062(2.38 s+1)^{2}}{(7.14 s+1)^{2}} & 0 & \frac{7.212(2.38 s+1)^{2}}{(1.43 s+1)^{2}} \\
\frac{0.038(11.36 s+1) e^{-5.99 s}}{22.22 s+1} & \frac{-1.213(11.36 s+1) e^{-1.94 s}}{(21.74 s+1)^{2}} & 0
\end{array}\right)
$$

Although the process is a 3x3 system, the complexity of the elements of (45) is as simple as that of the inverted decoupler elements for $2 \times 2$ processes. Three PI controllers are independently tuned for the corresponding decoupled processes $\mathrm{q}_{\mathrm{i}}(\mathrm{s})$ based on the IMC rules of Rivera [29]. Closed-loop time constants of 15, 20 and $18 \mathrm{~s}$ are specified in

\begin{tabular}{|c|c|c|c|c|c|c|c|c|c|c|c|}
\hline \multirow{2}{*}{ Method } & \multicolumn{6}{|c|}{ PI parameters } & \multirow[b]{2}{*}{$\mathrm{IAE}_{1}$} & \multirow[b]{2}{*}{$\mathrm{IAE}_{2}$} & \multirow[b]{2}{*}{$\mathrm{IAE}_{3}$} & \multirow{2}{*}{$\mu_{\mathrm{RS}}$} & \multirow[b]{2}{*}{$\mu_{\mathrm{RP}}$} \\
\hline & $K_{p 1}$ & $K_{p 2}$ & $K_{p 3}$ & $T_{i 1}$ & $T_{i 2}$ & $T_{i 3}$ & & & & & \\
\hline $\begin{array}{c}\text { Proposed } \\
\left(\mathrm{n}_{11}=\mathrm{e}^{-0.095}, \mathrm{n}_{33}=\mathrm{e}^{-0.26 \mathrm{~s}}\right)\end{array}$ & 2.25 & 0.77 & 0.07 & 67.1 & 5.1 & 12.3 & 15 & 21.3 & 22.9 & 0.28 & 0.86 \\
\hline Wang & - & - & - & - & - & - & 38.7 & 31 & 38.3 & 0.22 & 1.24 \\
\hline Liu & - & - & - & - & - & - & 17.6 & 25.1 & 22.9 & 0.23 & 0.77 \\
\hline $\begin{array}{c}\text { Lee } \\
\left(\lambda_{1}=15, \lambda_{2}=20, \lambda_{3}=18\right)\end{array}$ & 2.14 & -0.04 & 0.13 & 61.1 & 2.39 & 37.5 & 65.1 & 22.8 & 133 & 0.31 & 1.8 \\
\hline
\end{tabular}
each loop, respectively. The parameters are listed in Table 3.

Table 3 - PI parameters, performance and robustness indices for the different control methods for Example 2

The closed loop system responses (outputs and control signals) are shown in Figure 9. There are unit step changes at $t=0 \mathrm{~s}$ in the first reference, at $\mathrm{t}=333 \mathrm{~s}$ in the second one and at $\mathrm{t}=666 \mathrm{~s}$ in the third one.

For comparison, other control methodologies are also shown in the figure: the pure centralized control of Wang in [18], the analytical decoupling control of Liu in [30], and a multiloop PI controller. Some elements of the controllers of Wang, K(s), and Liu, $\mathrm{C}(\mathrm{s})$, are shown in (46) and (47), respectively. In them, all of the elements have a high order in the numerator and denominator, about third, fourth, fifth or sixth order plus time delay. Their higher complexity is clear in comparison with the elements of the 
proposed control in (45). The multiloop PI controller is tuned based on Lee's method [28], and using the same closed-loop time constants as the proposed control. According to the relative gain array (RGA), the chosen pairing has been $\mathrm{y}_{1}-\mathrm{u}_{1}, \mathrm{y}_{2}-\mathrm{u}_{3}$ and $\mathrm{y}_{3}-\mathrm{u}_{2}$. The PI parameters are collected in Table 3 with the performance and robustness indices of the different methodologies.

$$
\begin{aligned}
& k_{21}(s)=\frac{-69.82 s^{3}+10.68 s^{2}+s+0.01277}{1041 s^{3}+126.5 s^{2}+22.49 s} e^{-10.52 s}, \\
& 24260 s^{6}-10870 s^{5}+2651 s^{4}+ \\
& k_{12}(s)=\frac{-110 s^{3}+55.22 s^{2}+s+0.0006456}{-57140 s^{6}-17230 s^{5}-3031 s^{4}+} e^{-8.376 s}, \\
& -376.2 s^{3}-23.97 s^{2}-0.05722 s \\
& k_{33}(s)=\frac{-32.71 s^{3}-3.641 s^{2}+s+0.03188}{4964 s^{3}+411 s^{2}+102.3 s} \\
& c_{11}(s)=f_{1} \frac{14543 s^{2}+256.3578 s+0.5502}{(15 s+1)(438.7353 s+1)} e^{-0.09 s}, \\
& 4773900 s^{6}-6620600 s^{5}-3286200 s^{4} \\
& c_{12}(s)=f_{2} \frac{-532380 s^{3}-41045 s^{2}-523.1791 s-0.296}{(12 s+1)^{2}\left(611700 s^{4}+109510 s^{3}\right.} e^{-3.73 s} \text {, } \\
& \left.+12128 s^{2}+465.9313 s+1\right) \\
& c_{23}(s)=f_{3} \frac{16790 s^{3}+1582.9 s^{2}+39.2646 s+0.0885}{(18 s+1)\left(511.4853 s^{2}+440.0233 s+1\right)} \text {, } \\
& f_{1}=\frac{1}{1-\frac{e^{-0.8 s}}{15 s+1}}, f_{2}=\frac{1}{1-\frac{e^{-0.68 s}}{(12 s+1)^{2}}}, f_{3}=\frac{1}{1-\frac{e^{-1.85 s}}{18 s+1}}
\end{aligned}
$$

\section{INSERT HERE FIGURE 9}

The proposed design achieves perfect decoupling performance without overshoot or inverse response in the outputs; although the control signals are more oscillatory than in Wang's control. In addition, it obtains the smallest IAE. The response is quite similar than that of Liu's controller. Nevertheless, it is important to note that the complexity of the controller of Wang or Liu is much greater than that of the proposed control. In addition, these design procedures are more complex than that of the proposed inverted decoupling. The response of the multiloop controller is very good in the second loop; however, it shows important interactions in the other outputs.

In order to investigate the robustness of the controllers, a $\mu$-analysis is carried out as it was in the previous example. The selected weights are given by (48). Figure 10 shows the SSV for robust stability and robust performance for the different controllers. The robust stability is easily satisfied by all of controllers. The smallest values are achieved by the methods of Wang and Liu, although all values are very close. The robust performance analysis shows that the Liu's control and the proposed controller satisfy 
the robust performance condition (43). In spite of its high complexity, the performance of Wang's controller will deteriorate at frequency range of [0.01-0.1] rad/s, where a peak appears. The multiloop PI controller has a good robust stability; however, due to the strong interactions, it shows the worst robust performance at frequencies below 0.1 $\mathrm{rad} / \mathrm{s}$.

$$
\begin{aligned}
& W_{I}(s)=W_{I}(s) \cdot I=\frac{(2 s+0.2)}{s+1} \cdot I \\
& W_{P}(s)=W_{P}(s) \cdot I=\frac{(s / 2.75+0.025)}{s} \cdot I
\end{aligned}
$$

\section{INSERT HERE FIGURE 10}

\subsection{Example 4x4: HVAC process}

This interactive $4 \mathrm{x} 4$ process is the experimental centralized HVAC system of four rooms that appears in [31]. Air temperature control for four rooms is a complicated multivariable control problem because there are interactions among the air flow rates to the different rooms. The air temperature in the four rooms is controlled by regulating the position of four variable-air-volume (VAV) dampers. The $4 \times 4$ model is:

$$
\mathrm{G}_{\mathrm{H}}(\mathrm{s})=\left(\begin{array}{ccccc}
\frac{-0.098 \cdot e^{-17 s}}{122 s+1} & \frac{-0.036 \cdot e^{-27 s}}{149 s+1} & \frac{-0.014 \cdot e^{-32 s}}{158 s+1} & \frac{-0.017 \cdot e^{-30 s}}{155 s+1} \\
\frac{-0.043 \cdot e^{-25 s}}{147 s+1} & \frac{-0.092 \cdot e^{-16 s}}{130 s+1} & \frac{-0.011 \cdot e^{-33 s}}{156 s+1} & \frac{-0.012 \cdot e^{-34 s}}{157 s+1} \\
\frac{-0.012 \cdot e^{-31 s}}{153 s+1} & \frac{-0.016 \cdot e^{-34 s}}{151 s+1} & \frac{-0.102 \cdot e^{-16 s}}{118 s+1} & \frac{-0.033 \cdot e^{-26 s}}{146 s+1} \\
\frac{-0.013 \cdot e^{-32 s}}{156 s+1} & \frac{-0.015 \cdot e^{-31 s}}{159 s+1} & \frac{-0.029 \cdot e^{-25 s}}{144 s+1} & \frac{-0.108 \cdot e^{-18 s}}{128 s+1}
\end{array}\right)
$$

In [31], using the normalized decoupling control system design rules, an apparent decoupled process (50) is proposed. Then, a normalized decoupling network is designed using the ETF method [14]. By selecting a gain margin of 5 and a phase margin of $2 \pi / 5$ rad in all of the loops, a diagonal PI controller is designed. The parameters are collected in Table 4.

$$
\mathrm{Q}_{\mathrm{H}}(\mathrm{s})=\operatorname{diag}\left\{\frac{e^{-21.82 s}}{113.83 s+1}, \frac{e^{-21.32 s}}{121.37 s+1}, \frac{e^{-22.21 s}}{113.9 s+1}, \frac{e^{-23.12 s}}{123.55 s+1}\right\}
$$

In process (49), the only configuration for inverted decoupling that achieves realizability without adding extra dynamics is configuration 1-2-3-4. Using this configuration, the proposed extended approach of inverted decoupling is applied in such a way that the same apparent process (50) is specified in (19) and (20), resulting in the following Dd and Do matrices:

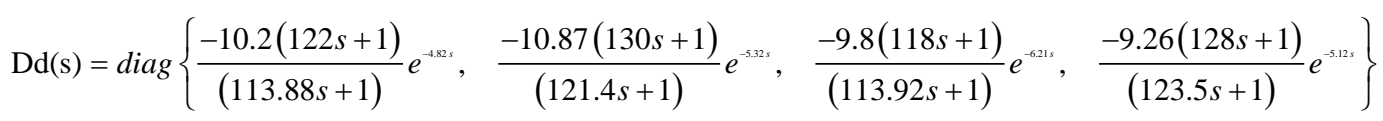


$\operatorname{Do}(\mathrm{s})=\left(\begin{array}{cccc}0 & \frac{(4.098 s+0.036)}{149 s+1} e^{-5.18 s} & \frac{(1.594 s+0.014)}{158 s+1} e^{-10.2 s} & \frac{(1.935 s+0.017)}{155 s+1} e^{-8.18 s} \\ \frac{(5.219 s+0.043)}{147 s+1} e^{-3.68 s} & 0 & \frac{(1.335 s+0.011)}{156 s+1} e^{-1.7 s} & \frac{(1.456 s+0.012)}{157 s+1} e^{-12.7 s} \\ \frac{(1.367 s+0.012)}{153 s+1} e^{-8.79 s} & \frac{(1.822 s+0.016)}{151 s+1} e^{-11.8 s} & 0 & \frac{(3.759 s+0.033)}{146 s+1} e^{-3.79 s} \\ \frac{(1.606 s+0.013)}{156 s+1} e^{-8.88 s} & \frac{(1.853 s+0.015)}{159 s+1} e^{-7.88 s} & \frac{(3.583 s+0.029)}{144 s+1} e^{-1.88 s} & 0\end{array}\right)$

In order to achieve the same performance obtained with the normalized decoupling in [31] and because the apparent decoupled process is practically identical, the same PI parameters are used in the proposed control. Figure 11 shows the closed loop system response (outputs and control signals) of the designed inverted decoupling control in comparison with that of the normalized decoupling of [31]. A multiloop PI controller based on the Lee's method [28] is also shown.

\begin{tabular}{|c|c|c|c|c|c|c|c|c|c|c|c|c|c|c|}
\hline \multirow[b]{2}{*}{ Method } & \multicolumn{8}{|c|}{ PI parameters } & \multirow{2}{*}{$\mathrm{IAE}_{1}$} & \multirow{2}{*}{$\mathrm{IAE}_{2}$} & \multirow{2}{*}{$\mathrm{IAE}_{3}$} & \multirow[b]{2}{*}{$\mathrm{IAE}_{4}$} & \multirow[b]{2}{*}{$\mu_{\mathrm{RS}}$} & \multirow[b]{2}{*}{$\mu_{\mathrm{RP}}$} \\
\hline & $K_{p 1}$ & $K_{p 2}$ & $K_{p 3}$ & $K_{p 4}$ & $T_{i 1}$ & $T_{i 2}$ & $T_{i 3}$ & $T_{i 4}$ & & & & & & \\
\hline Proposed & 1.64 & 1.79 & 1.61 & 1.68 & 69.4 & 67.8 & 70.7 & 73.5 & 80.4 & 78.1 & 81.7 & 85.1 & 0.22 & 0.97 \\
\hline $\begin{array}{l}\text { Normalized } \\
\text { Decoupling }\end{array}$ & 1.64 & 1.79 & 1.61 & 1.68 & 69.4 & 67.8 & 70.7 & 73.5 & 87.2 & 84.5 & 87.9 & 90.7 & 0.24 & 1.04 \\
\hline $\begin{array}{c}\text { Lee } \\
\left(\lambda_{\mathrm{i}}=45, \forall i\right)\end{array}$ & - & $\begin{array}{c}- \\
23.5\end{array}$ & $\begin{array}{c}- \\
19.3\end{array}$ & $\begin{array}{c}- \\
19.2\end{array}$ & 101.8 & 108.3 & 108.2 & 117.4 & 99.9 & 99.1 & 95.2 & 95.8 & 0.41 & 1.22 \\
\hline
\end{tabular}

Table 4 - PI parameters, performance and robustness indices for the different control methods for Example 3

\section{INSERT HERE FIGURE 11}

There is a unit step change in each reference every 500 seconds, and at $t=2000 \mathrm{~s}$, there is a unit step in all process inputs at the same time as the input disturbance.

The performances of the different methodologies are quite similar with the same settling time. However, the proposed control achieves perfect decoupling, while the multiloop control presents some interactions. Normalized decoupling also shows some little interactions. The IAE indices are collected in Table 4. They are very similar; however, the proposed control obtains the smallest ones because it achieves perfect decoupling.

Although the complexities of both decouplers are practically the same, another advantage of the proposed methodology over normalized decoupling is its direct method of carrying out the decoupler network design. In the normalized decoupling design, the procedure is slightly more complex with the calculation of the normalized gain matrix, the RGA, the RNGA and the RARTA.

Using the weights in (53), a $\mu$-analysis in presence of diagonal multiplicative input uncertainty shows that the three controllers have similar robust stability and robust performance. Results are depicted in Figure 12 and the peak values are collected in 
Table 4. The multiloop control has a worse robust performance in a small range of frequencies due to the interactions.

$$
\begin{aligned}
& \mathrm{W}_{\mathrm{I}}(\mathrm{s})=\mathrm{W}_{\mathrm{I}}(\mathrm{s}) \cdot \mathrm{I}=\frac{(10 \mathrm{~s}+0.2)}{5 \mathrm{~s}+1} \cdot \mathrm{I} \\
& \mathrm{W}_{\mathrm{P}}(\mathrm{s})=\mathrm{W}_{\mathrm{P}}(\mathrm{s}) \cdot \mathrm{I}=\frac{(\mathrm{s} / 2+0.008)}{\mathrm{s}} \cdot \mathrm{I}
\end{aligned}
$$

\section{INSERT HERE FIGURE 12}

\subsection{Experimental process: quadruple tank system}

The experimental process is a quadruple tank plant [32] in the lab of the Computer Science Department of the University of Córdoba. The outputs are the level of the lower tanks inside the range of [0-35] cm, and the inputs are the flow references of the secondary control loops that regulate the operation of the pumps, in the range of [0-200] $\mathrm{cm}^{3} / \mathrm{s}$. The plant was configured in order to show interaction problems without having multivariable RHP zeros, and then, the process was identified around the operation point $\mathrm{h}=[2020] \mathrm{cm}$ and $\mathrm{u}=[135135] \mathrm{cm}^{3} / \mathrm{s}$. The resultant model is given by (54), and it has a RGA of 2.29 .

$$
\mathrm{G}_{\mathrm{Q}}(\mathrm{s})=\left(\begin{array}{cc}
\frac{0.3284}{184.5 s+1} & \frac{0.2454}{(184.5 s+1)(535.1 s+1)} \\
\frac{0.2457}{(185 s+1)(503.2 s+1)} & \frac{0.3378}{185 s+1}
\end{array}\right)
$$

Due to relative degrees, configuration 1-2 must be chosen for realizability without adding extra dynamics. If the $\operatorname{Dd}(\mathrm{s})$ matrix is fixed to the unitary matrix, according to Table 1 , the apparent processes $\mathrm{q}_{1}(\mathrm{~s})$ and $\mathrm{q}_{2}(\mathrm{~s})$ are given by $\mathrm{g}_{11}(\mathrm{~s})$ and $\mathrm{g}_{22}(\mathrm{~s})$ of (54), and the other two decoupler elements of Do(s) are:

$$
\begin{aligned}
& \mathrm{do}_{12}=\frac{-\mathrm{g}_{12}}{\mathrm{~g}_{11}}=\frac{-2454}{3284(535.1 s+1)} \\
& \mathrm{do}_{21}=\frac{-\mathrm{g}_{21}}{\mathrm{~g}_{22}}=\frac{-2457}{3378(503.2 s+1)}
\end{aligned}
$$

After determining the decoupler elements, the parameters of a PI decentralized

\begin{tabular}{|c|c|c|c|c|c|c|c|c|}
\hline \multirow[b]{2}{*}{ Method } & \multicolumn{4}{|c|}{ PI parameters } & \multirow[b]{2}{*}{$\mathbf{I A E}_{1}$} & \multirow[b]{2}{*}{$\mathbf{I A E}_{2}$} & \multirow[b]{2}{*}{$\mu_{\mathrm{RS}}$} & \multirow[b]{2}{*}{$\mu_{\mathrm{RP}}$} \\
\hline & $K_{p 1}$ & $K_{p 2}$ & $T_{i 1}$ & $T_{i 2}$ & & & & \\
\hline Proposed & 4.68 & 4.56 & 184.4 & 185 & 1002 & 1.27 & 0.35 & 0.97 \\
\hline Decentralized & 4.68 & 4.56 & 184.5 & 185 & 1895 & 1.22 & 0.37 & 0.99 \\
\hline
\end{tabular}
controller are independently tuned for the corresponding $\mathrm{q}_{i}(\mathrm{~s})$ using the IMC tuning rules of [29]. A closed loop time constant of $120 \mathrm{~s}$ is specified in both loops to obtain a settling time of about $600 \mathrm{~s}$. The parameters of the two resulting PI controllers are shown in Table 5 with the performance and robustness indices. 
Table 5 - PI parameters and performance and robustness indices for a quadruple tank process

Figure 13 shows the resultant response of the closed loop system for a step of $5 \mathrm{~cm}$ in the references. For comparison, we also show the response of the decentralized controller obtained only with the PI controllers in Table 5, without the decoupler elements (55). Therefore, it is like two independent SISO controllers, one for $\mathrm{g}_{11}(\mathrm{~s})$ and the other for $\mathrm{g}_{22}(\mathrm{~s})$.

With inverted decoupling, a better response is achieved with a very good decoupling performance and a smaller settling time in both loops. The decentralized control reaches the references later, and the rejection of the interactions is very slow, and therefore, the corresponding IAE indices are greater than those of the proposed control. Using the model in (54), a $\mu$-analysis in presence of diagonal multiplicative input uncertainty is carried out. The robust stability and robust performance indices in Table 5 for both controllers are quite similar.

\section{INSERT HERE FIGURE 13}

\section{Conclusions}

A new generalized approach of the inverted decoupling technique for nxn processes has been developed in this work. The problem is approached from a compact matricial formulation, and we demonstrate that the transfer functions of the decoupler elements and the decoupled equivalent open loop process have the same complexity, regardless of the system size. This is a great advantage over other conventional decoupling methods. In addition, the methodology allows for more flexibility in choosing the decoupled apparent processes, and since they are usually very simple, the tuning of decentralized controllers is much easier. Therefore, it is concluded that the proposed methodology has important design advantages that make it very easy to apply when the process does not have multivariable RHP zeros.

A study of all possible configurations for inverted decoupling was carried out, and the realizability conditions for applying this decoupling method were expounded. The expressions of inverted decoupling for $2 \times 2$ and $3 \times 3$ processes are shown in more detail. The methodology is illustrated with three simulation examples of different sizes (2x2, $3 x 3$ and $4 x 4$ ). PI decentralized controllers were used to control the apparent decoupled processes. The system time responses are compared with other works, demonstrating that this decoupling achieves similar or better performance. Additionally, an experimental lab process of four tanks was used to verify the effectiveness of this methodology.

\section{Acknowledgments}

This work was supported by the Spanish CICYT under Grant DPI 2007-62052. This support is very gratefully acknowledged. Moreover, J. Garrido expresses appreciation for the FPU fellowship (Ref. AP2006-01049) from the Spanish Ministry of Education.

\section{References}


[1] F. C. Shinskey, Sistemas de control de procesos. Aplicación, diseño y sintonización. Tomo 1, McGrawhill, 1996.

[2] F. Vázquez, F. Morilla, S. Dormido, An iterative method for tuning decentralized PID controllers, Proceeding of the $14^{\text {th }}$ IFAC World Congress (1999) 491-496.

[3] F. Vázquez, Diseño de controladores PID para sistemas MIMO con control descentralizado, PhD Thesis, UNED, 2001.

[4] Q. W. Wang, B. Zou, T. Lee, Q. Bi, Auto-tuning of Multivariable PID Controllers from Decentralized Feedback, Automatica 33 (1997) 319-330.

[5] Q. W. Wang, Y. Zhang, M. S. Chiu, Decoupling internal model control for multivariable systems with multiple time delays, Chem. Eng. Sci. 57 (2002) 115-124.

[6] Q. W. Wang, Y. Zhang, M. S. Chiu, None-interacting control design for multivariable industrial processes, J. Process Control 13 (2003) 253-265.

[7] Q. Xiong, W. J. Cai, M. J. He, Equivalent transfer function method for PI/PID controller design of MIMO processes, J. Process Control 17 (2007) 665-673.

[8] F. Morilla, F. Vázquez, J. Garrido, Centralized PID Control by Decoupling for TITO Processes, Proceedings of 13th IEEE International Conference on Emerging Technologies and Factory Automation (2008) 1318-1325.

[9] J. Garrido, F. Morilla, F. Vázquez, Centralized PID Control by Decoupling of a Boiler-Turbine Unit, 10th European Control Conference, Budapest (2009) 4007-4012.

[10] K. Waller, Decoupling in distillation, AIChE J. 20 (1974) 592-594.

[11] H. L. Wade, Inverted decoupling: a neglected technique, ISA Trans. 36 (1997) 3-10.

[12] P. Nordfeldt, T. Hägglund, Decoupler and PID controller design of TITO systems, J. Process Control 16, (2006) 923-936.

[13] S. Tavakoli, I. Griffin, P. J. Fleming, Tuning of decentralised PI (PID) control for TITO processes, Control Eng. Pract. 14 (2006) 1069-1080.

[14] W. J. Cai, W. Ni, M. J. He, C. Y. Ni, Normalized Decoupling - A new approach for MIMO process control system design, Ind. Eng. Chem. Res. 47 (2008) 7347-7356.

[15] J. Maciejowski, Multivariable Feedback Design, Addison-Wesley, 1989.

[16] S. Skogestad, I. Postlethwaite, Multivariable Feedback Control: Analysis and Design, John Wiley \& sons, 1996.

[17] G. C. Goodwin, S. F. Graebe, M. E. Salgado, Control System Design, Prentice-Hall, 2001.

[18] Q. W. Wang, Decoupling Control, Lecture Notes in Control and Information Sciences, 285, Springer-Verlag, 2003.

[19] K. J. Åström, K. H. Johansson, Q. W. Wang, Design of decoupled PI controller for two-by-two systems, IEE Proc. Contr. Theor. Appl. 149 (2002) 74-81.

[20] F. Vázquez, F. Morilla, Tuning decentralized PID controllers for MIMO systems with decouplers, Proceeding of the 15th World Congress of the IFAC, Barcelona, Spain (2002).

[21] E. Gagnon, A. Pomerleau, A. Desbiens, Simplified, ideal or inverted decoupling?, ISA Trans. 37 (1998) 265-276.

[22] J. Zheng, G. Guo, Y. Wang, Feedforward decoupling control design for dual-actuator system in hard disk drives, IEEE Trans. Magn. 40 (2004) 2080-2082.

[23] P. Chen, W. Zhang, Improvement on an inverted decoupling technique for a class of stable linear multivariable processes, ISA Trans. 46 (2007) 199-210.

[24] R. Garduno, K. W. Lee, Compensation of control-loop interaction for power plant wide-range operation, Control Eng. Pract. 13, (2005) 1475-1487.

[25] T. Hägglund, K. J. Åström, Revisiting the Ziegler-Nichols step response method for PID control, J. Process Control 14 (2004) 635-650.

[26] B. D. Tyreus, Multivariable control system design for an industrial distillation column, Ind. Eng. Chem. Process Des. Dev. 18 (1979) 177-182.

[27] A. O’Dwyer, PI and PID controller tuning design for processes with delay to achieve constant gain and phase margin for all values of delay, Proceedings of the Irish Signals and Systems Conference (2001) 96-100.

[28] M. Lee, K. Lee, C. Kim, J. Lee, Analytical design of multiloop PID controllers for desired closed-loop responses, AIChE J. 50 (2004) 1631-1635.

[29] D. E. Rivera, M. Morari, S. Skogestad, Internal Model Control. 4. PID controller design, Ind. Eng. Chem. Process Des. Dev. 25, (1986) 252-265.

[30] T. Liu, W. Zhang, F. Gao, Analytical decoupling control strategy using a unity feedback control structure for MIMO processes with time delays, J. Process Control 17 (2007) 173-186. 
[31] Y. Shen, W. Cai, S. Li, Normalized decoupling control for high-dimensional MIMO processes for application in room temperature control HVAC systems, Control Eng. Pract. 18 (2010) 652664.

[32] K. H. Johansson, The quadruple-tank process: a multivariable laboratory process with an adjustable zero, IEEE Trans. Control Syst. Technol. 8 (2000) 456-465. 


\section{Table Captions}

Table 1 - Cases of $2 \times 2$ inverted decoupling with two unitary elements (configuration 12).

Table 2 - Different control methods for Example 1 with performance and robustness indices.

Table 3 - PI parameters, performance and robustness indices for the different control methods for Example 2.

Table 4 - PI parameters, performance and robustness indices for the different control methods for Example 3.

Table 5 - PI parameters and performance and robustness indices for a quadruple tank process. 


\begin{tabular}{|c|c|c|}
\hline & Decoupler elements & Decoupled process \\
\hline Case 1 & $\begin{array}{c}\mathrm{dd}_{11}=\mathrm{dd}_{22}=1 \\
\mathrm{do}_{12}=\frac{-\mathrm{g}_{12}}{\mathrm{~g}_{11}}, \quad \mathrm{do}_{21}=\frac{-\mathrm{g}_{21}}{\mathrm{~g}_{22}}\end{array}$ & $\mathrm{Q}=\left(\begin{array}{cc}\mathrm{g}_{11} & 0 \\
0 & \mathrm{~g}_{22}\end{array}\right)$ \\
\hline Case 2 & $\begin{array}{c}\mathrm{dd}_{11}=\mathrm{do}_{21}=1 \\
\mathrm{do}_{12}=\frac{-\mathrm{g}_{12}}{\mathrm{~g}_{11}}, \quad \mathrm{dd}_{22}=\frac{-\mathrm{g}_{21}}{\mathrm{~g}_{22}}\end{array}$ & $Q=\left(\begin{array}{cc}g_{11} & 0 \\
0 & -g_{21}\end{array}\right)$ \\
\hline Case 3 & $\begin{array}{c}\mathrm{do}_{12}=\mathrm{do}_{21}=1 \\
\mathrm{dd}_{11}=\frac{-\mathrm{g}_{12}}{\mathrm{~g}_{11}}, \quad \mathrm{dd}_{22}=\frac{-\mathrm{g}_{21}}{\mathrm{~g}_{22}}\end{array}$ & $\mathrm{Q}=\left(\begin{array}{cc}-\mathrm{g}_{12} & 0 \\
0 & -\mathrm{g}_{21}\end{array}\right)$ \\
\hline Case 4 & $\begin{array}{c}\mathrm{do}_{12}=\mathrm{dd}_{22}=1 \\
\mathrm{dd}_{11}=\frac{-\mathrm{g}_{12}}{\mathrm{~g}_{11}}, \quad \mathrm{do}_{21}=\frac{-\mathrm{g}_{21}}{\mathrm{~g}_{22}}\end{array}$ & $\mathrm{Q}=\left(\begin{array}{cc}-\mathrm{g}_{12} & 0 \\
0 & \mathrm{~g}_{22}\end{array}\right)$ \\
\hline
\end{tabular}




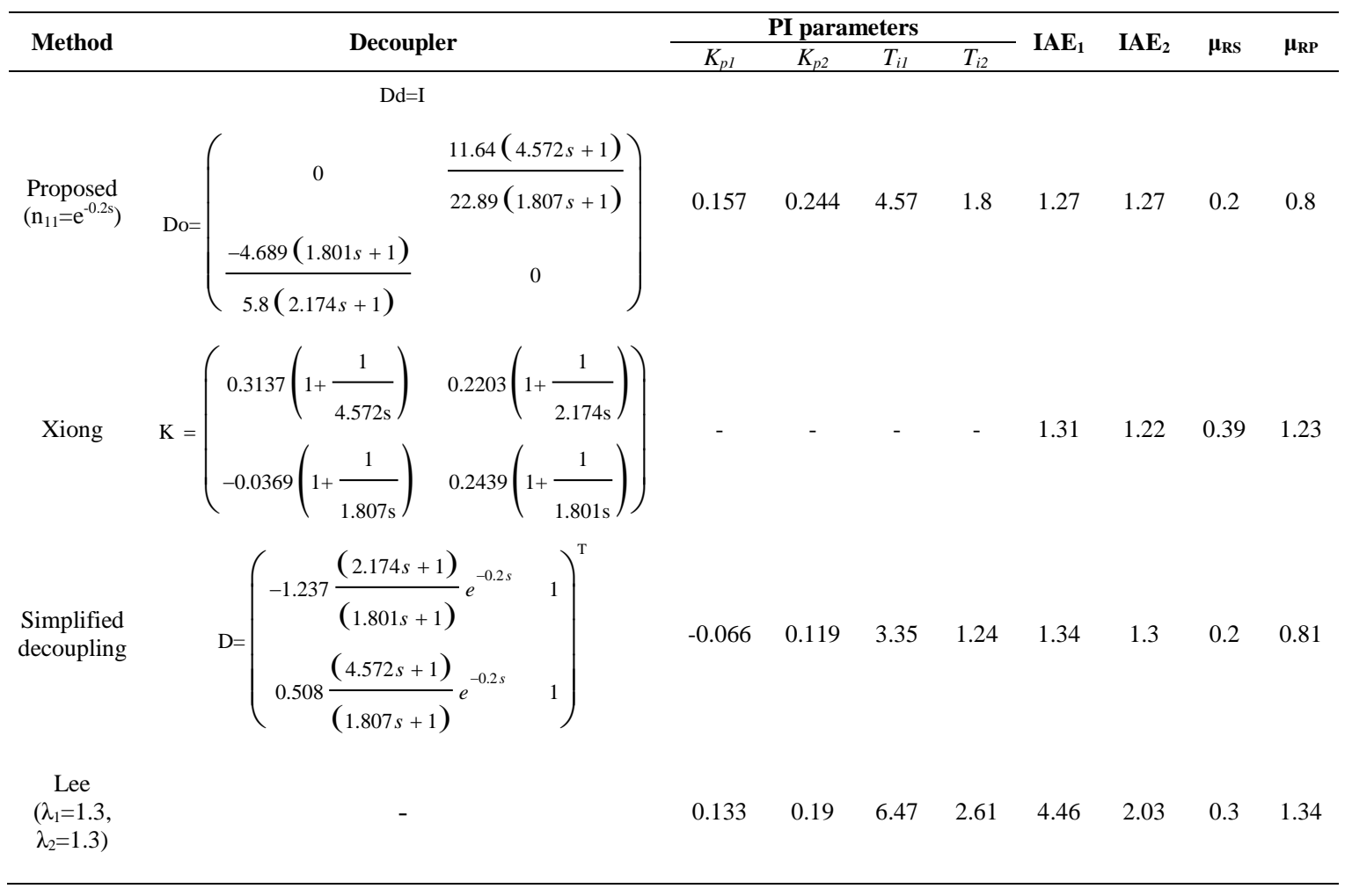




\begin{tabular}{|c|c|c|c|c|c|c|c|c|c|c|c|}
\hline \multirow{2}{*}{ Method } & \multicolumn{6}{|c|}{ PI parameters } & \multirow{2}{*}{$\mathbf{I A E}_{\mathbf{1}}$} & \multirow{2}{*}{$\mathbf{I A E}_{2}$} & \multirow{2}{*}{$\mathbf{I A E}_{\mathbf{3}}$} & \multirow[b]{2}{*}{$\mu_{\mathrm{RS}}$} & \multirow[b]{2}{*}{$\mu_{\mathrm{RP}}$} \\
\hline & $K_{p l}$ & $K_{p 2}$ & $K_{p 3}$ & $T_{i l}$ & $T_{i 2}$ & $T_{i 3}$ & & & & & \\
\hline $\begin{array}{c}\text { Proposed } \\
\left(\mathrm{n}_{11}=\mathrm{e}^{-0.09 \mathrm{~s}}, \mathrm{n}_{33}=\mathrm{e}^{-0.26 \mathrm{~s}}\right)\end{array}$ & 2.25 & 0.77 & 0.07 & 67.1 & 5.1 & 12.3 & 15 & 21.3 & 22.9 & 0.28 & 0.86 \\
\hline Wang & - & - & - & - & - & - & 38.7 & 31 & 38.3 & 0.22 & 1.24 \\
\hline Liu & - & - & - & - & - & - & 17.6 & 25.1 & 22.9 & 0.23 & 0.77 \\
\hline $\begin{array}{c}\text { Lee } \\
\left(\lambda_{1}=15, \lambda_{2}=20, \lambda_{3}=18\right)\end{array}$ & 2.14 & -0.04 & 0.13 & 61.1 & 2.39 & 37.5 & 65.1 & 22.8 & 133 & 0.31 & 1.8 \\
\hline
\end{tabular}




\begin{tabular}{|c|c|c|c|c|c|c|c|c|c|c|c|c|c|c|}
\hline \multirow{2}{*}{ Method } & \multicolumn{8}{|c|}{ PI parameters } & \multirow[b]{2}{*}{$\mathbf{I A E}_{1}$} & \multirow[b]{2}{*}{$\mathbf{I A E}_{2}$} & \multirow[b]{2}{*}{$\mathbf{I A E}_{3}$} & \multirow[b]{2}{*}{$\mathbf{I A E}_{4}$} & \multirow[b]{2}{*}{$\mu_{\mathrm{RS}}$} & \multirow[b]{2}{*}{$\mu_{\mathrm{RP}}$} \\
\hline & $K_{p l}$ & $K_{p 2}$ & $K_{p 3}$ & $K_{p 4}$ & $T_{i l}$ & $T_{i 2}$ & $T_{i 3}$ & $T_{i 4}$ & & & & & & \\
\hline Proposed & 1.64 & 1.79 & 1.61 & 1.68 & 69.4 & 67.8 & 70.7 & 73.5 & 80.4 & 78.1 & 81.7 & 85.1 & 0.22 & 0.97 \\
\hline $\begin{array}{l}\text { Normalized } \\
\text { Decoupling }\end{array}$ & 1.64 & 1.79 & 1.61 & 1.68 & 69.4 & 67.8 & 70.7 & 73.5 & 87.2 & 84.5 & 87.9 & 90.7 & 0.24 & 1.04 \\
\hline $\begin{array}{c}\text { Lee } \\
\left(\lambda_{\mathrm{i}}=45, \forall i\right)\end{array}$ & -20.5 & -23.5 & -19.3 & -19.2 & 101.8 & 108.3 & 108.2 & 117.4 & 99.9 & 99.1 & 95.2 & 95.8 & 0.41 & 1.22 \\
\hline
\end{tabular}




\begin{tabular}{|c|c|c|c|c|c|c|c|c|}
\hline \multirow{2}{*}{ Method } & \multicolumn{4}{|c|}{ PI parameters } & \multirow{2}{*}{$\mathbf{I A E}_{1}$} & \multirow{2}{*}{$\mathbf{I A E}_{2}$} & \multirow{2}{*}{$\mu_{\mathrm{RS}}$} & \multirow{2}{*}{$\mu_{\mathrm{RP}}$} \\
\hline & $K_{p 1}$ & $K_{p 2}$ & $T_{i 1}$ & $T_{i 2}$ & & & & \\
\hline Proposed & 4.68 & 4.56 & 184.4 & 185 & 1002 & 1.27 & 0.35 & 0.97 \\
\hline Decentralized & 4.68 & 4.56 & 184.5 & 185 & 1895 & 1.22 & 0.37 & 0.99 \\
\hline
\end{tabular}




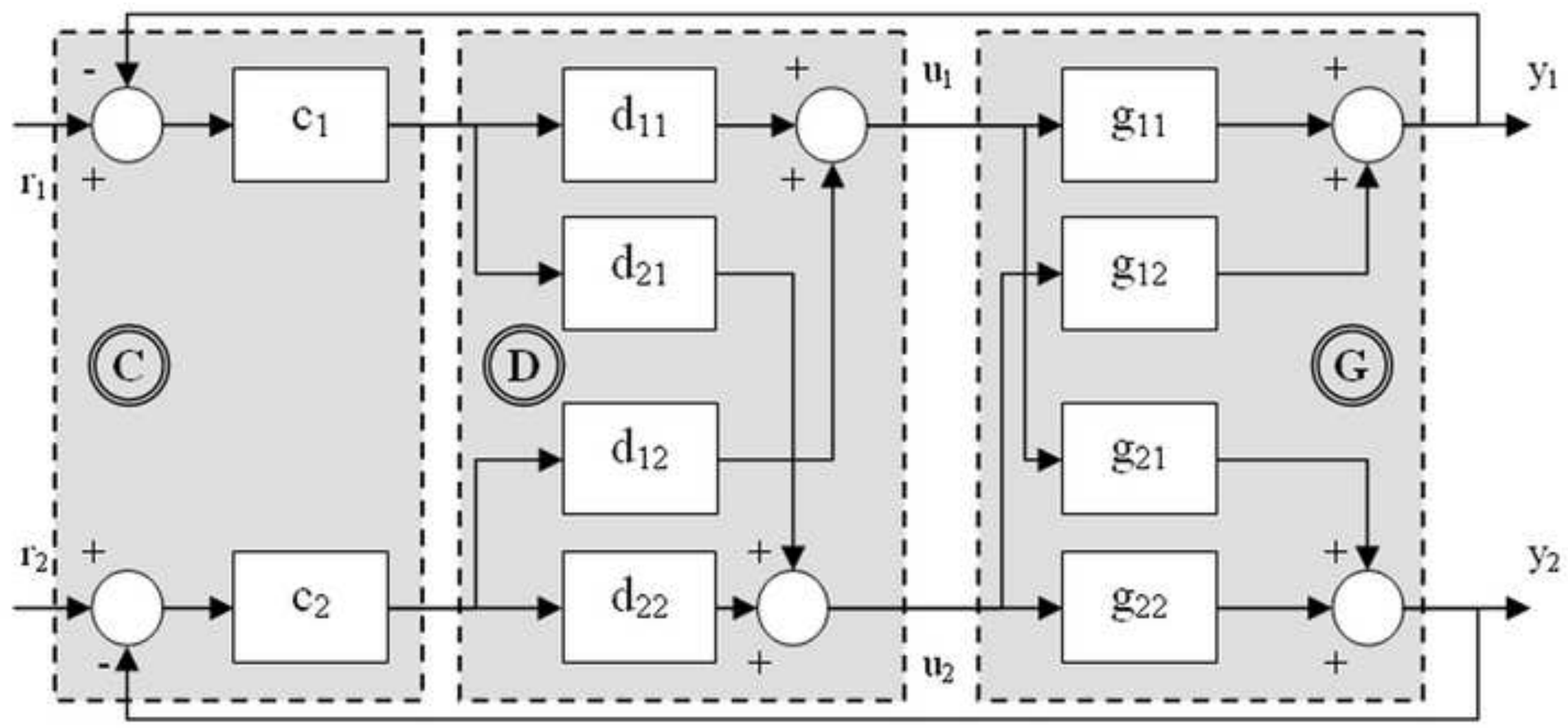




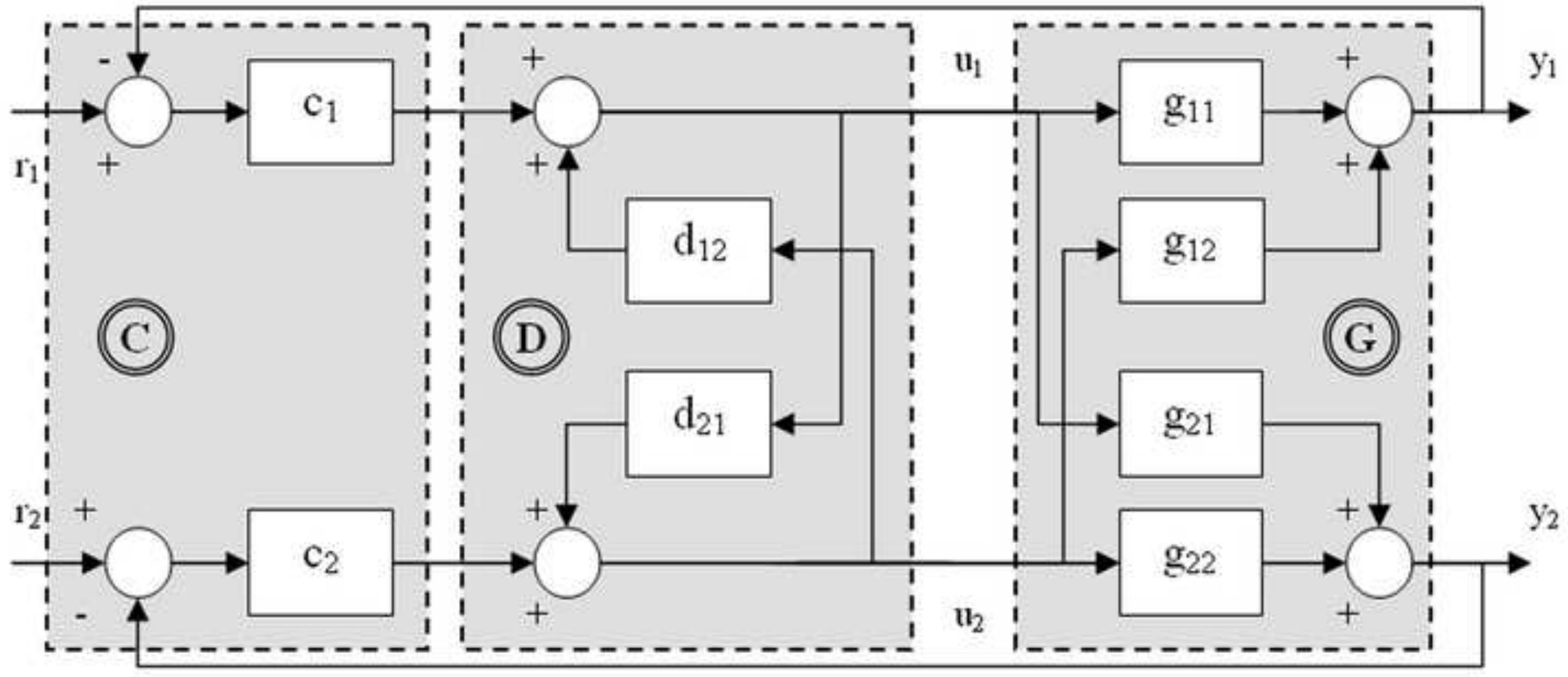




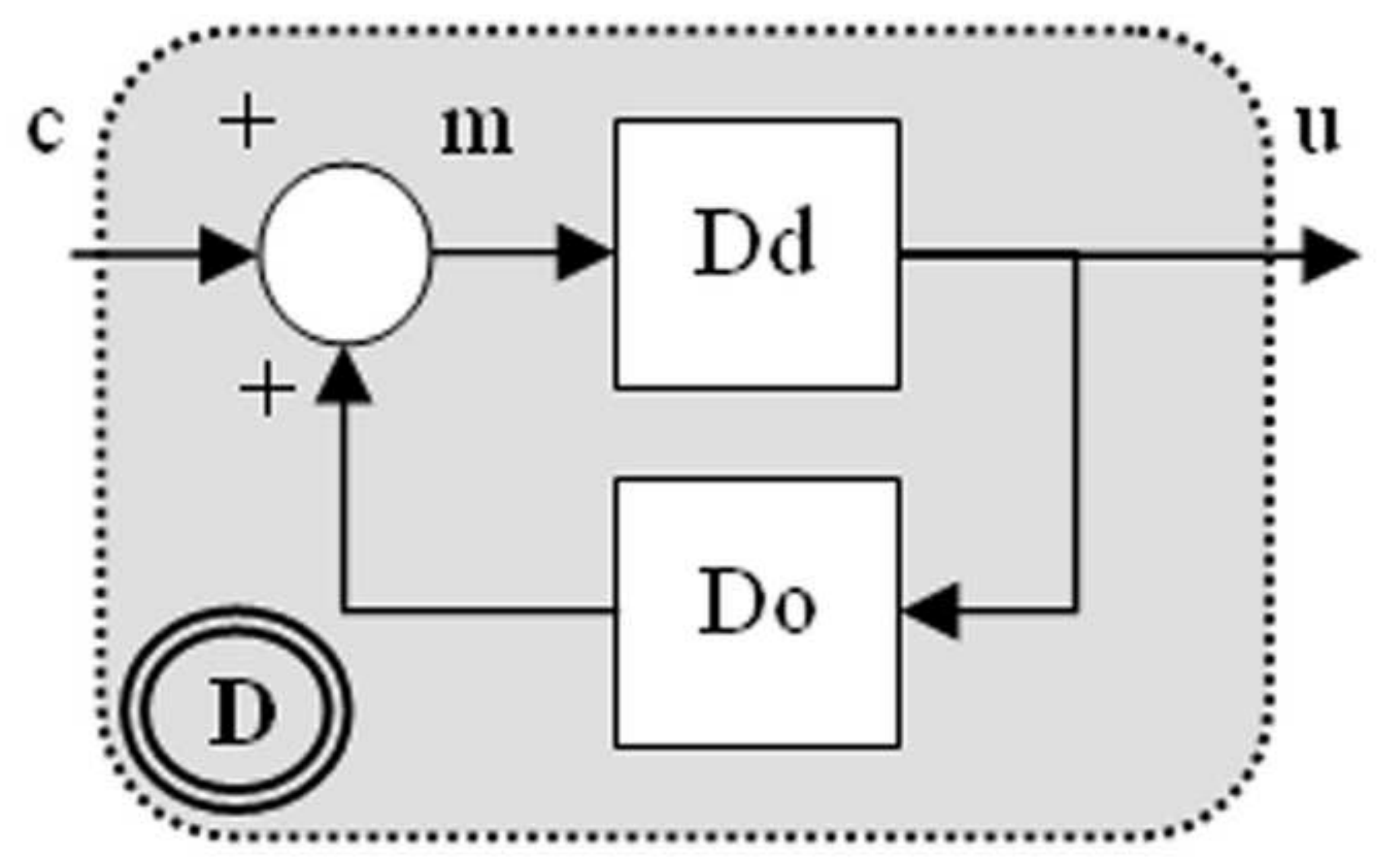




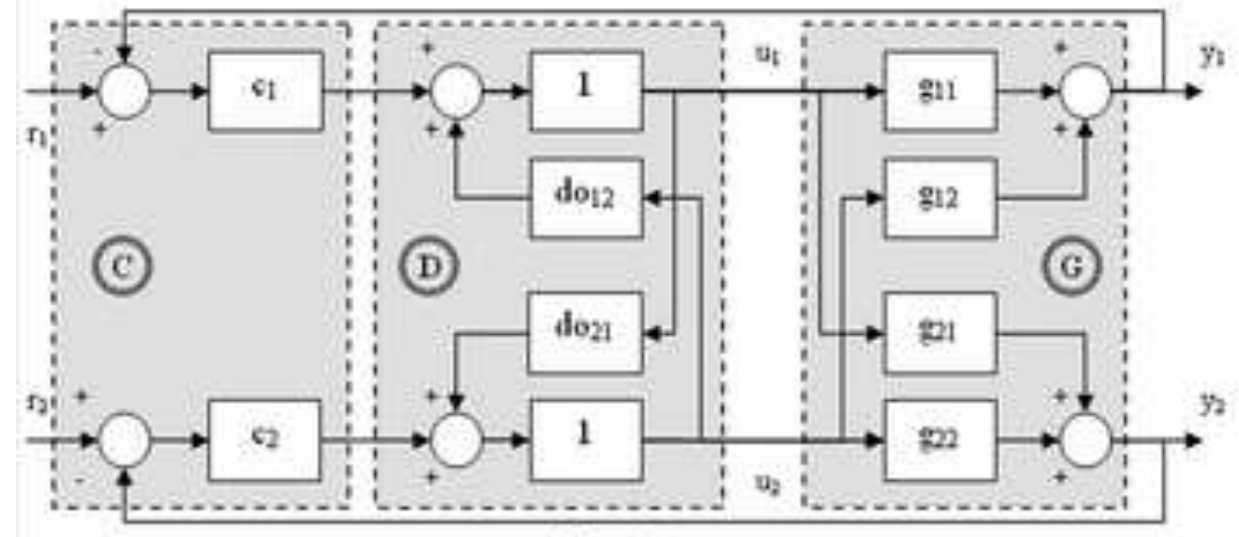

\section{Case 1}

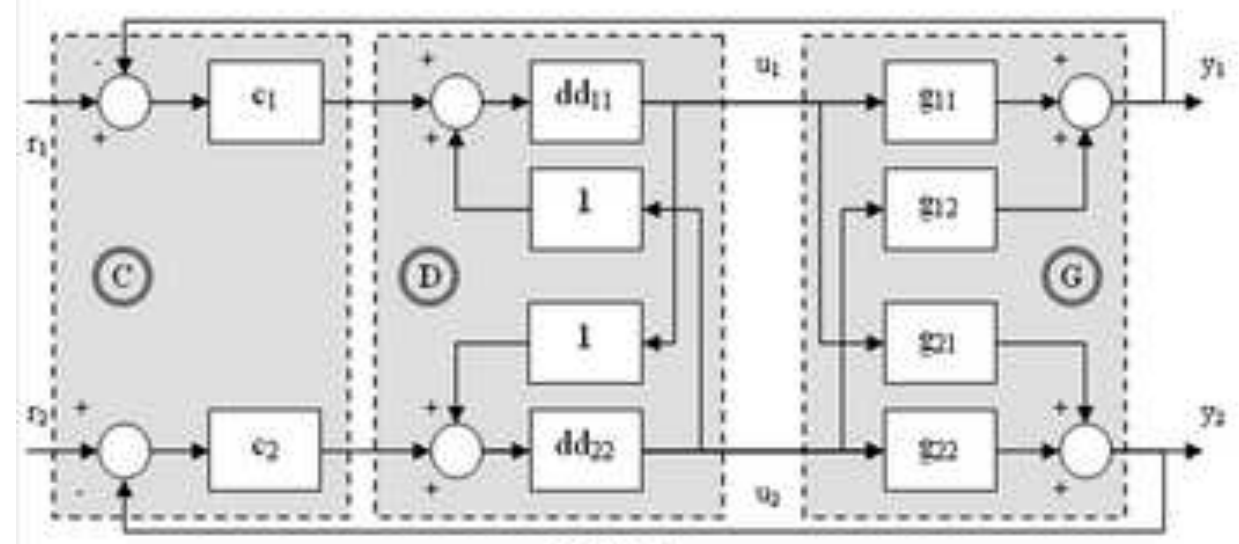

Case 3
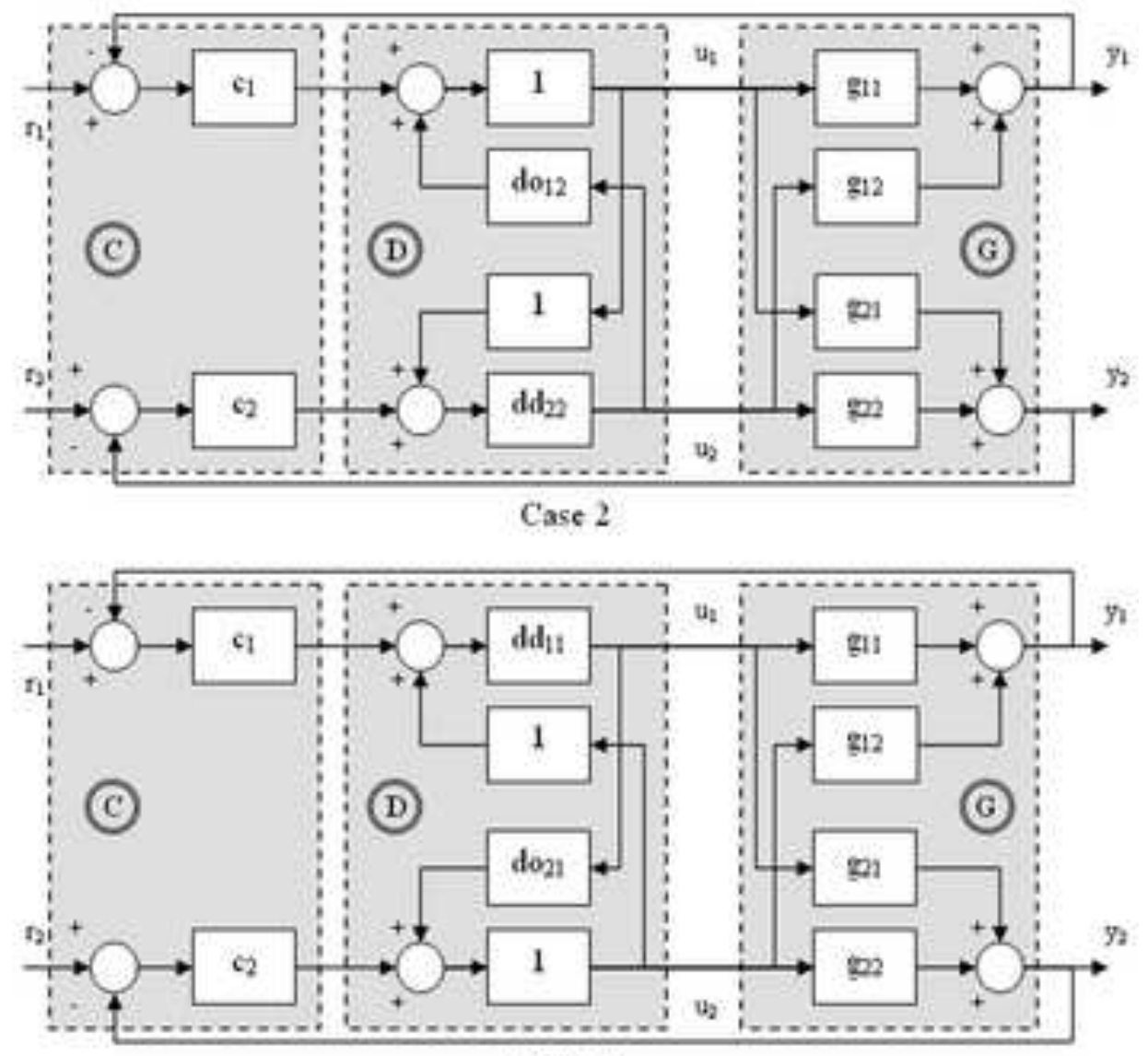

Case 4 
Figure 5. Step response of system (21).

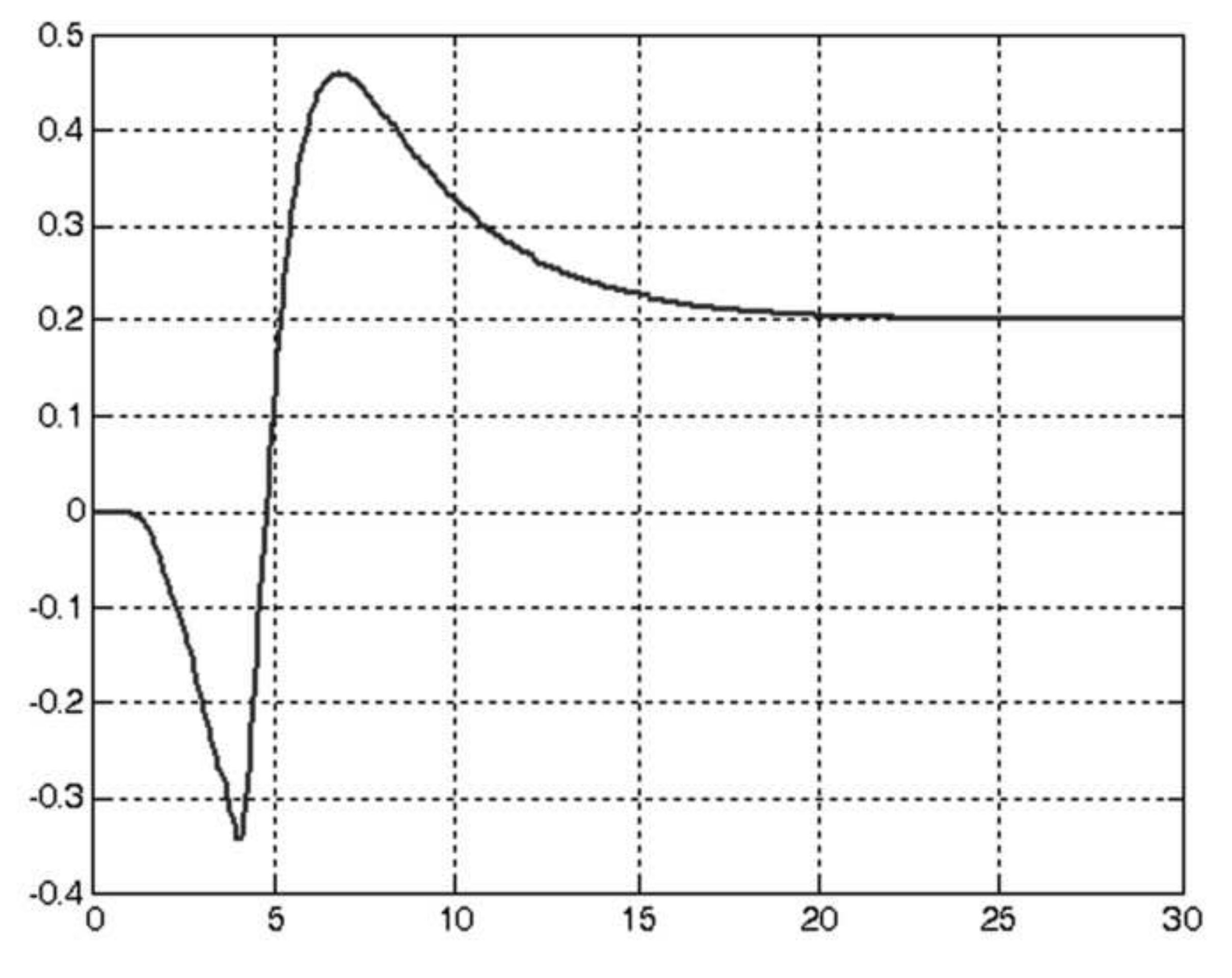

.



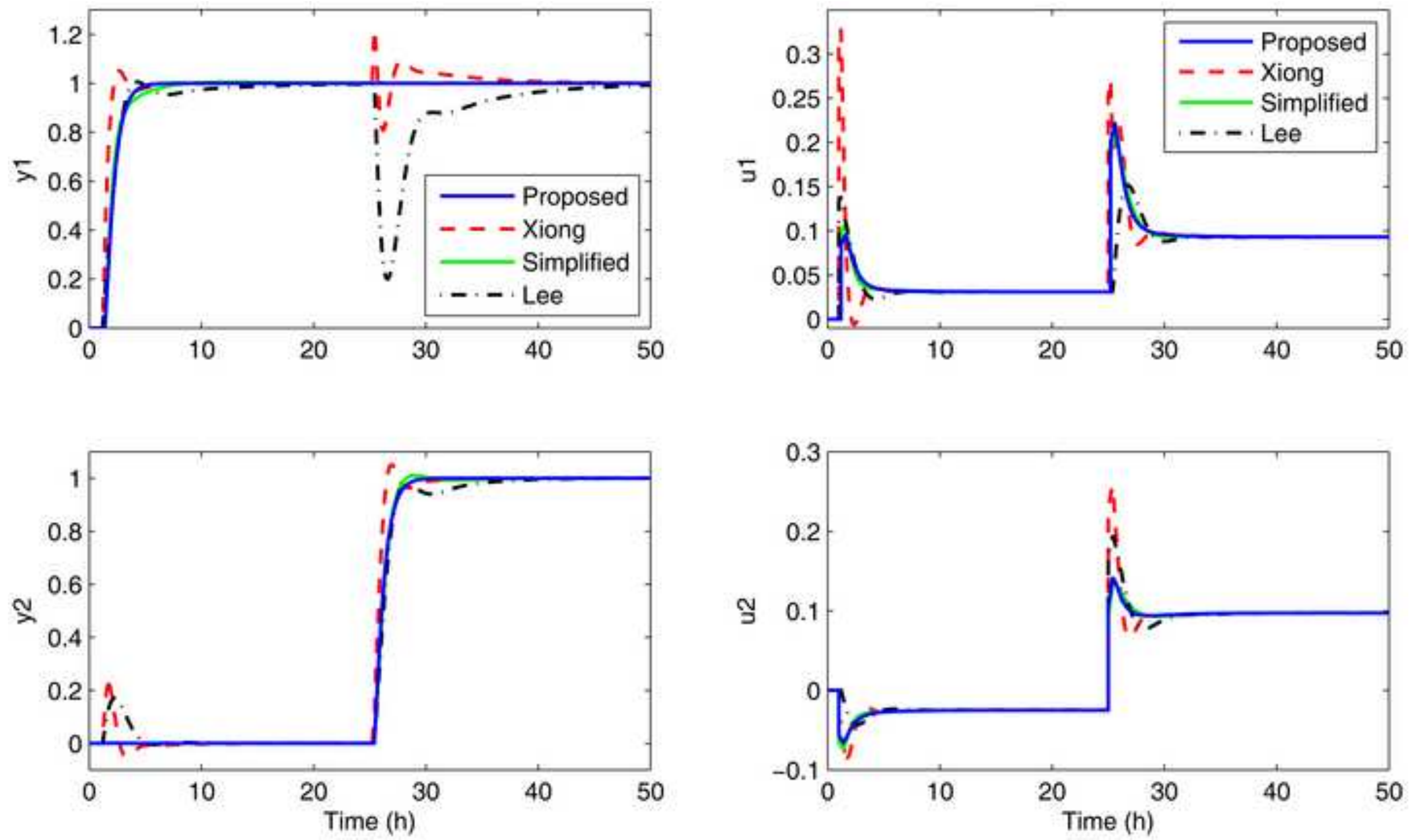


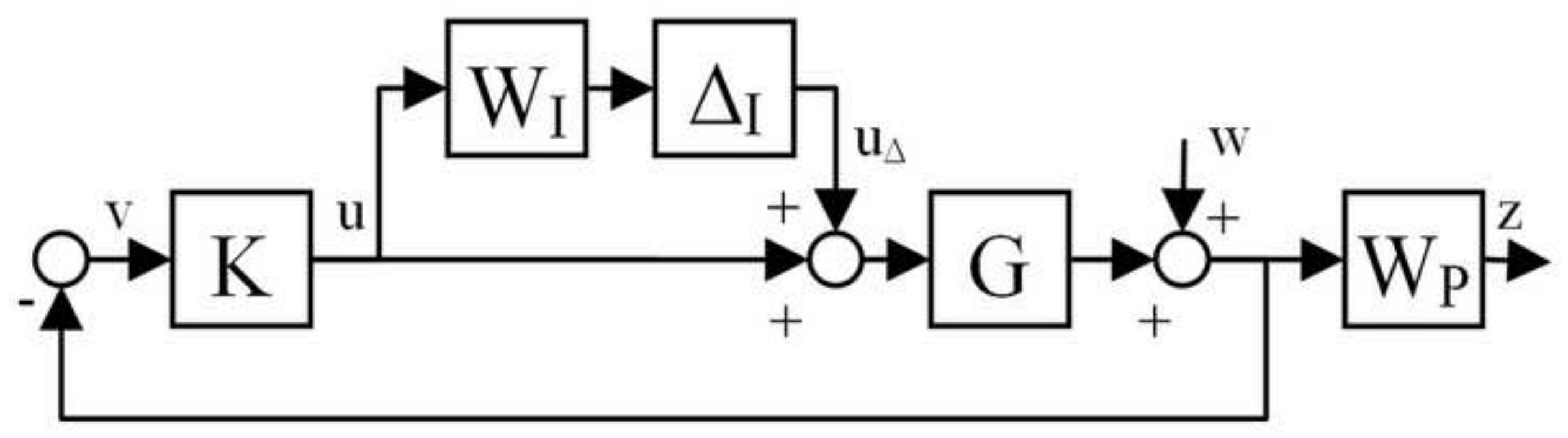



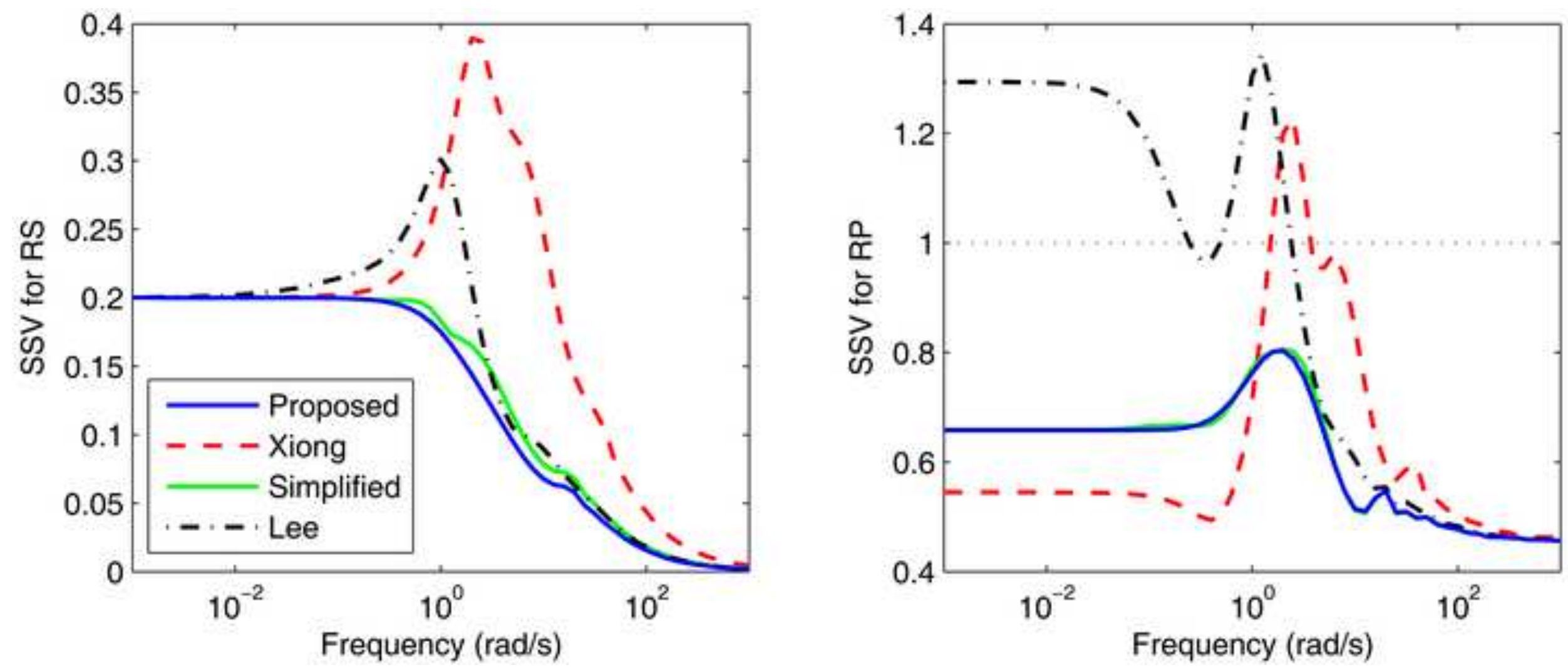
Figure 9. Outputs and control signals in example 2
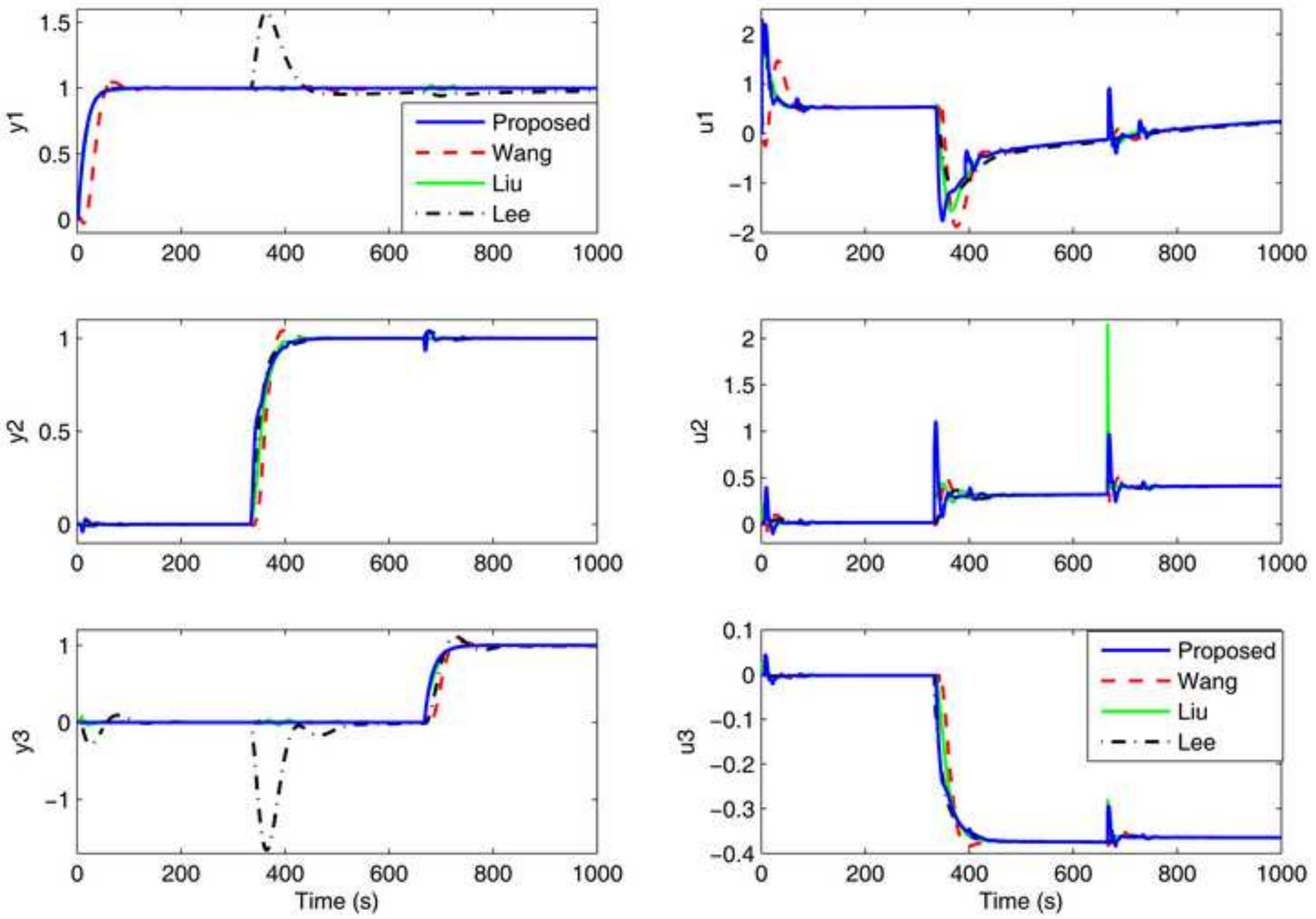

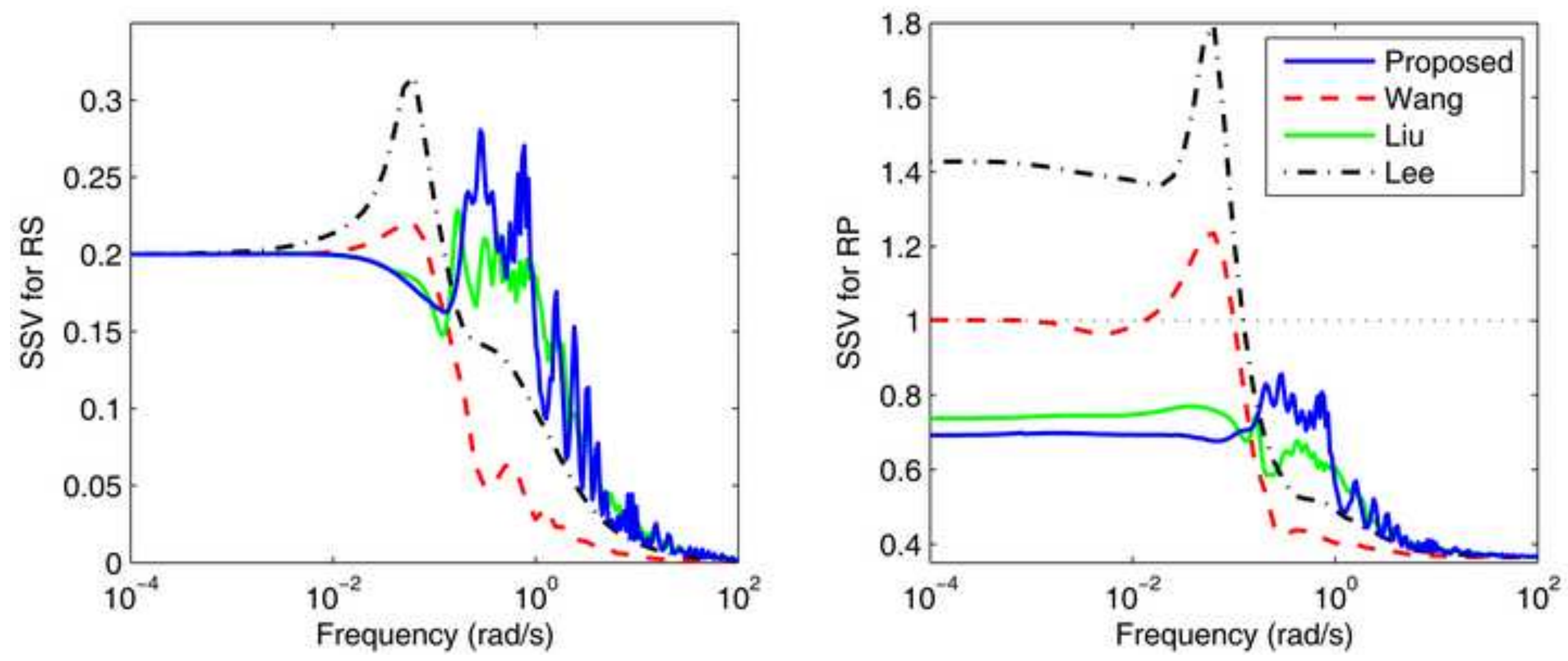

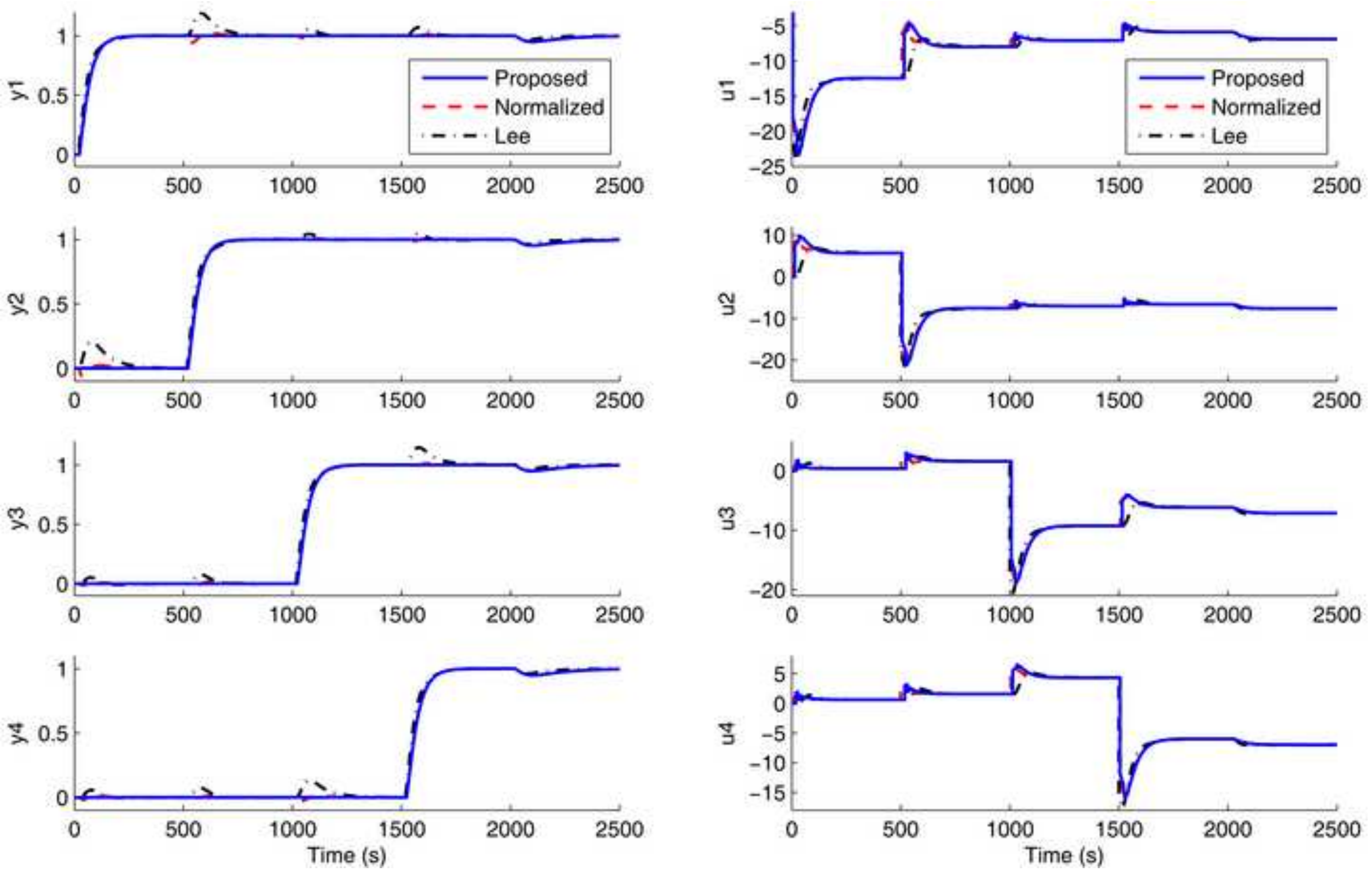

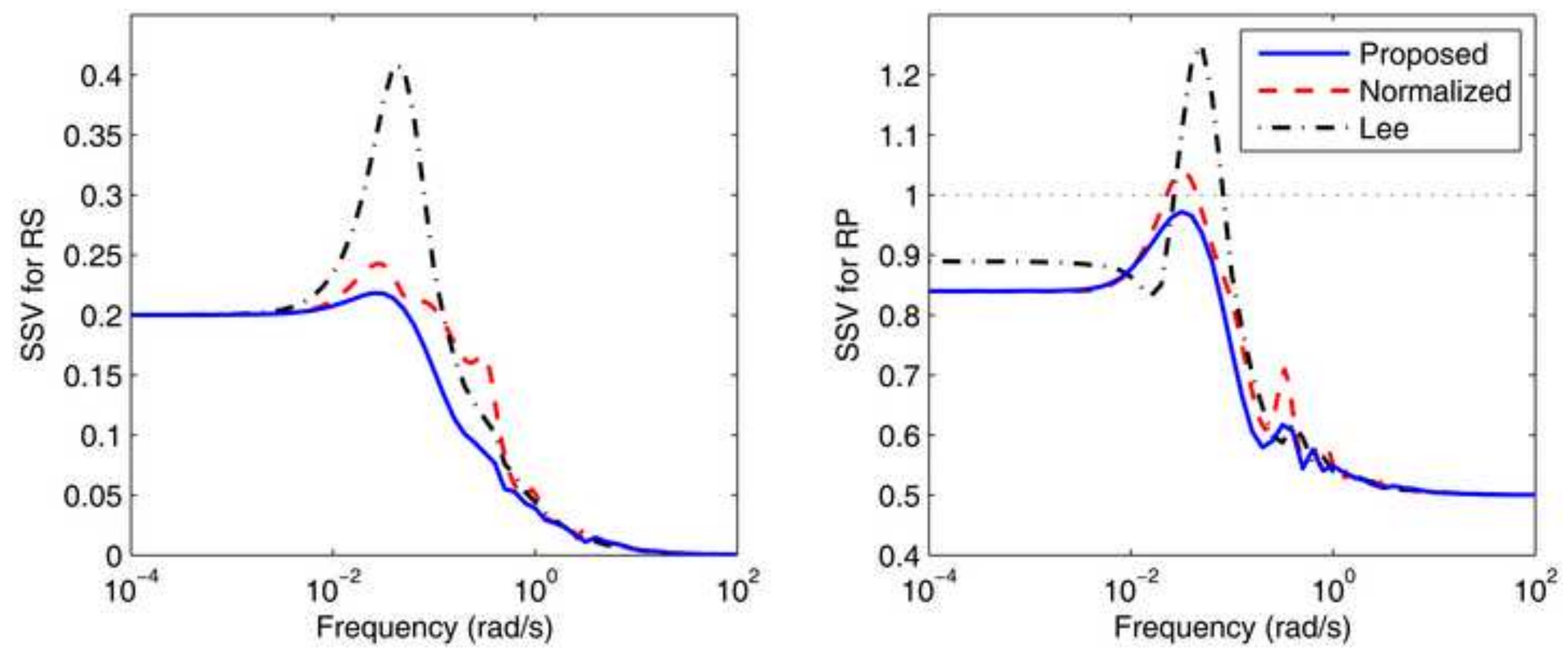

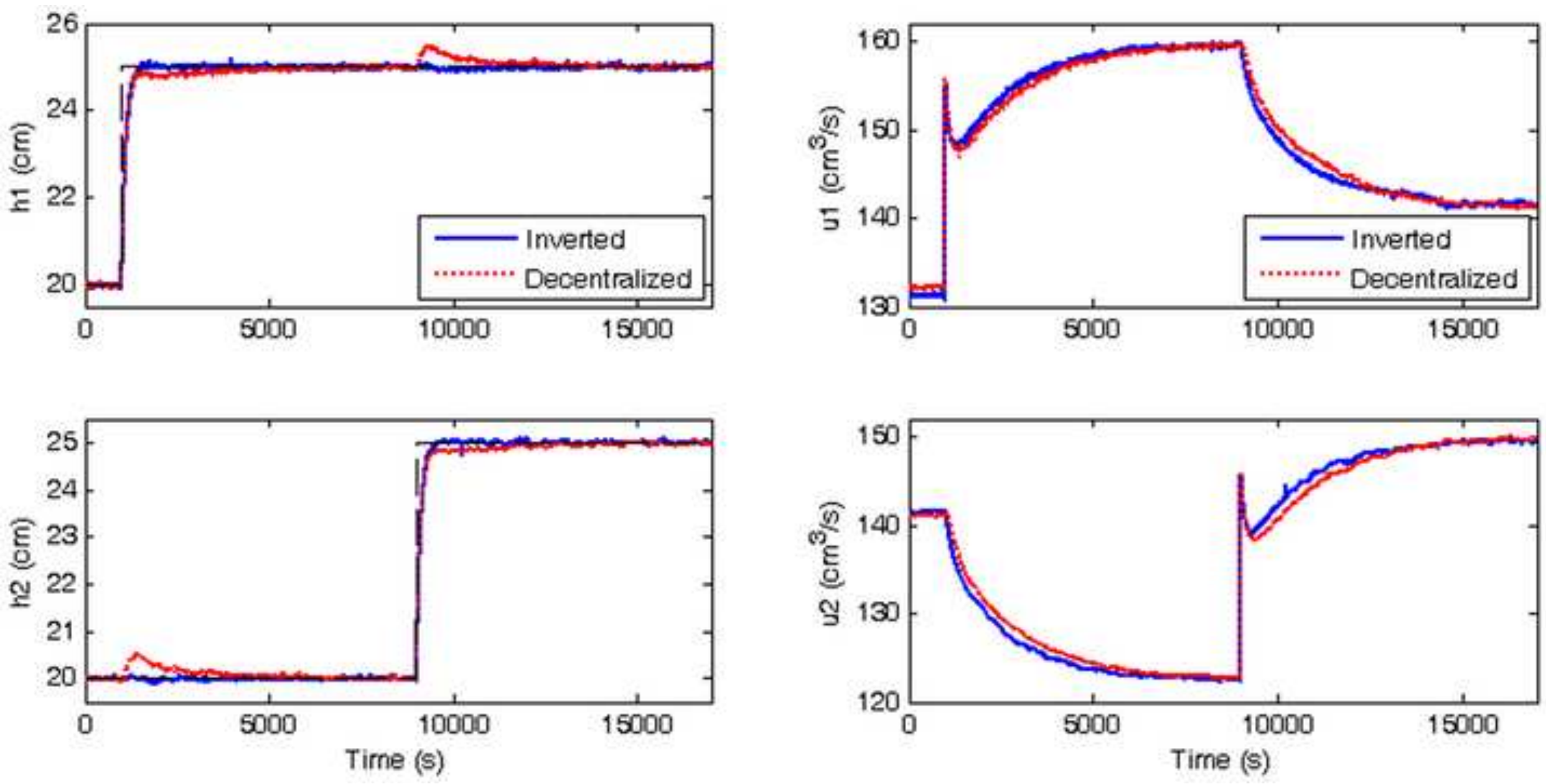